\title{
SECOND ORDER THETA FUNCTIONS AND VECTOR BUNDLES OVER JACOBI VARIETIES
}

\author{
DAVID S. YUEN
}

\begin{abstract}
We consider the Picard vector bundles defined over Jacobi varieties. The rank $g+1$ Picard bundle imbeds in the rank $2^{g}$ Clifford bundle, so the second order theta functions, viewed appropriately, span the dual of the Picard bundle over each fiber. We prove a result on the minimum number of such second order theta functions required to span the whole bundle at each point. We give an application of using these functions to describe subvarieties of the Jacobian. There follow comments on which functions we could use, and generalizations to higher order theta functions.
\end{abstract}

\section{INTRODUCTION}

Over the Jacobi variety of a compact Riemann surface, there are the naturally occurring Picard vector bundles. These bundles are derived by considering sufficiently high symmetric products of the curve, which map to the Jacobi variety. They reflect many essential properties of the Jacobi variety and of the curve. However, there is of yet no explicit description of these Picard bundles. They were initially investigated by Kempf [12], and Mattuck [16, 17]. Gunning has shown [6] that the rank $g+1$ Picard bundle sits inside the rank $2^{g}$ Clifford bundle, representing the well-known transformation properties of second order theta function under half periods. One aim of this paper is to find in some sense a smaller explicitly describable bundle in which this Picard bundle sits. We prove a result that there exist $2 g+1$ second order theta functions that span the dual of this Picard bundle, and that this is the minimum number required. This implies that the pullback of this Picard bundle under an isogeny of the Jacobi variety sits inside a rank $2 g+1$ bundle which is the rank $2 g+1$ trivial bundle tensored with a line bundle. This result generalizes to the the rank $(d-1) g+1$ Picard bundle. There follow some comments on whether we can say which $2 g+1$ second order theta functions we can use.

Received by the editors May 1, 1989.

1980 Mathematics Subject Classification (1985 Revision). Primary 14H40; Secondary 14K25.

This work is based in part on the author's doctoral dissertation at Princeton University, and was supported in part by a National Science Foundation fellowship. 
At this time I would like to thank Professor Robert Gunning for his indispensable help and kindness.

\section{Notations}

In this section, we will fix some notations regarding marked Riemann surfaces and their Jacobians, vector bundles and factors of automorphy, theta functions, and Chern classes.

Marked Riemann surfaces. Let $M$ be a marked compact Riemann surface of genus $g>1$, represented as the quotient $M=\widetilde{M} / \Gamma$ of its universal covering space $\widetilde{M}$ by the group $\Gamma$ of covering translations. As in [7], a marking is viewed as a choice of a base point $z_{0} \in \widetilde{M}$ and a set of canonical generators $A_{1}, \ldots, A_{g}, B_{1}, \ldots, B_{g}$ of the group $\Gamma$. Then we have a canonical basis of holomorphic abelian differentials $\omega_{1}, \ldots, \omega_{g}$. Denote by $w_{i}$ the corresponding abelian integrals, viewed as functions on $\widetilde{M}$, that is, $w_{i}(z)=\int_{z_{0}}^{z} \omega_{i}$. They are normalized by $w_{i}\left(z_{0}\right)=0$. For each $T \in \Gamma$, denote the period of $\omega_{i}$ with respect to $T$ by $\omega_{i}(T)$, so $w_{i}(T z)=w_{i}(z)+\omega_{i}(T)$ for all $z \in \widetilde{M}$. The periods are normalized by $\omega_{i}\left(A_{j}\right)=\delta_{j}^{i}$. Denote by $w$ the vector valued function with components $w_{i}$ so that $w: \widetilde{M} \rightarrow \mathbf{C}^{g}$, and also denote by $\omega(T)$ the vector in $\mathbf{C}^{g}$ with components $\omega_{i}(T)$. We have the Abel-Jacobi map

$$
w: M \rightarrow J=\mathbf{C}^{g} / \mathscr{L},
$$

where $J=J(M)$ is the Jacobian of $M$ with $\mathscr{L}=\omega(\Gamma)=\mathbf{Z}^{g}+\Omega Z^{g}$ and $\Omega=$ the $g \times g$ matrix $\left(\omega_{i j}\right)$ where $\omega_{i j}=\omega_{i}\left(B_{j}\right)$. The Jacobian is a principally polarized variety with an irreducible theta locus.

Vector bundles. Holomorphic vector bundles over $M$ will be described by factors of automorphy. As in [7], a rank $n$ factor of automorphy $\sigma$ is a holomorphic map $\sigma: \widetilde{M} \times \Gamma \rightarrow \operatorname{GL}(n, \mathrm{C})$ such that $\sigma(z, S T)=\sigma(T z, S) \sigma(z, T)$ for all $z \in \widetilde{M}$ and $S, T \in \Gamma$. Precisely, the vector bundle associated to $\sigma$ has total space

$$
\begin{gathered}
E(\sigma)=\underset{M}{ } \times \mathbf{C}^{n} /(\sim) \\
\downarrow \\
M
\end{gathered}
$$

with the equivalence relation being $(z, c) \sim(T z, \sigma(z, T) c)$ for $z \in \widetilde{M}$, $T \in \Gamma$, and $c \in \mathbf{C}^{n}$. Two factors of automorphy $\sigma_{1}, \sigma_{2}$ describe the same holomorphic vector bundle precisely when there exists a holomorphic map $F: \widetilde{M} \rightarrow \mathrm{GL}(n, \mathbf{C})$ such that $F(T z) \sigma_{2}(z, T)=\sigma_{1}(z, T) F(z)$ for all $z \in \widetilde{M}$ and $T \in \Gamma$. This gives the notion of equivalent factors of automorphy. More generally, a map $F: \sigma_{1} \rightarrow \sigma_{2}$ of two vector bundles $\sigma_{1}, \sigma_{2}$ of ranks $n_{1}$, $n_{2}$, respectively is given by a map $F: \widetilde{M} \rightarrow \operatorname{Mat}\left(n_{2} \times n_{1}, \mathrm{C}\right)$ which satisfies $F(T z) \sigma_{1}(z, T)=\sigma_{2}(z, T) F(z)$. In particular, a section of a vector bundle $\sigma$, 
which is a map $s: \mathscr{I} \rightarrow \sigma$, is described by a relatively automorphic function $s: \widetilde{M} \rightarrow \mathbf{C}^{n}$ for which $s(T z)=\sigma(z, T) s(z)$ for all $z \in \widetilde{M}$ and $T \in \Gamma$. Such sections form a vector space denoted by $\Gamma(M, \sigma)$. We will denote the trivial vector bundle of rank $n$ by $\mathscr{I}_{n}$.

As in [6], let $\zeta$ denote the line bundle on $M$ associated to the divisor consisting of the base point of the marking. For any $t \in \mathbf{C}^{g}, \rho_{t}$ will denote the flat line bundle on $M$ with factor of automorphy $\rho_{t}\left(A_{j}\right)=1$ and $\rho_{t}\left(B_{j}\right)=\exp 2 \pi i t_{j}$.

Similarly, vector bundles over the Jacobian $J=\mathbf{C}^{g} / \mathscr{L}$ will also be described by factors of automorphy, $\sigma: \mathbf{C}^{g} \times \mathscr{L} \rightarrow \mathrm{GL}(n, \mathbf{C})$ such that $\sigma\left(w, \lambda_{1}+\lambda_{2}\right)=$ $\sigma\left(w+\lambda_{1}, \lambda_{2}\right) \sigma\left(w, \lambda_{1}\right)$ for all $w \in \mathbf{C}^{g}$ and $\lambda_{1}, \lambda_{2} \in \mathscr{L}$. The total space of the associated vector bundle is

$$
\begin{gathered}
E(\sigma)=\mathbf{C}^{g} \times \mathbf{C}^{n} /(\sim) \\
\downarrow \\
J
\end{gathered}
$$

with the equivalence relation being $(w, c) \sim(w+\lambda, \sigma(w, \lambda) c)$ for $w \in \mathbf{C}^{g}$, $\lambda \in \mathscr{L}$, and $c \in \mathbf{C}^{n}$. For any $t \in \mathbf{C}^{g}$, denote also by $\rho_{t}$ the flat line bundle with factor of automorphy $\rho_{t}(\lambda)=\exp 2 \pi i^{t} q t$ for any $\lambda=p+\Omega q \in \mathscr{L}$.

Via the imbedding $w: M \hookrightarrow J$, any vector bundle $\sigma$ on $J$ induces by restriction a vector bundle $w^{*} \sigma$ on $M$. We know $w^{*} \rho_{t}=\rho_{t}$ and $w^{*} \xi=\rho_{r} \zeta^{g}$ where $r \in \mathbf{C}^{g}$ is the Riemann point associated to the marking, and $\xi$ is the theta line bundle defined in the following paragraphs.

Theta functions. For our period matrix $\Omega$, and in general any symmetric matrix with positive definite imaginary part, we define the first order theta function with characteristics as

$$
\theta[\nu \mid \tau](w ; \Omega)=\sum_{n \in \mathbf{Z}^{8}} \exp 2 \pi i\left(\frac{1}{2}^{t}(n+\nu) \Omega(n+\nu)+{ }^{t}(n+\nu)(w+\tau)\right),
$$

where $\nu, \tau, w \in \mathbf{C}^{g}$. The simple theta function is $\theta(w)=\theta[0 \mid 0](w)$, and its zero locus is the theta locus denoted by $\Theta \subset J$. The $d$ th order theta function with characteristics is defined as

$$
\theta_{d}[\nu \mid \tau](w: \Omega)=\theta\left[\frac{\nu}{d} \mid \tau\right](d w ; d \Omega) .
$$

For $\nu \in \mathbf{Z}^{g}$, this function of $w$ is a relatively automorphic function for $\rho_{-\tau} \xi^{d}$, where $\xi$ is the theta factor of automorphy with

$$
\xi(w, \lambda)=\exp -2 \pi i^{t} q\left(w+\frac{1}{2} \Omega q\right) .
$$

In fact $\theta_{d}[\nu \mid \tau](w)$, for $\nu \in \mathbf{Z}^{g} / d \mathbf{Z}^{g}$ form a basis of $\Gamma\left(J, \rho_{-\tau} \xi^{d}\right)$. Denote

$$
\vec{\theta}_{d}[-\tau](w)=\left(\theta_{d}[\nu \mid-\tau](w)\right)_{\nu}, \quad \text { the vector } \in \mathbf{C}^{d^{8}} .
$$

Then the elements of $\Gamma\left(J, \rho_{\tau} \xi^{d}\right)$ are exactly ${ }^{t} c \cdot \vec{\theta}_{d}[-\tau](w)$, for $c \in \mathbf{C}^{d^{8}}$. This gives a canonical identification

$$
\Gamma\left(J, \rho_{\tau} \xi^{d}\right)=\mathbf{C}^{d^{g} t} c \cdot \vec{\theta}_{d}[-\tau](w) \leftrightarrow c .
$$


Define a new notation

$$
\theta_{d}\langle c \mid \tau\rangle(w ; \Omega) \stackrel{\text { def }}{=} c \cdot \vec{\theta}_{d}[\tau](w ; \Omega),
$$

for $c \in \mathbf{C}^{d^{g}}, \tau \in \mathbf{C}^{g}$ and $w \in \mathbf{C}^{g}$. Note that in this notation, $\theta_{d}\langle c \mid \tau\rangle(w ; \Omega)$ is linear in $c$.

Chern classes. To every rank $n$ vector bundle $\sigma$ over $J$ is associated its total Chern class

$$
c(\sigma)=1+c_{1}+\cdots+c_{n} \in H^{*}(J, \mathbf{Z}),
$$

where $c_{i}(\sigma) \in H^{2 i}(J, \mathbf{Z})$. Definitions and basic properties can be found in [11].

We now fix some notation regarding the cohomology ring of $J$. Since an abelian variety of complex dimension $g$ is topologically a product of $2 g$ circles, its cohomology ring is merely an exterior algebra on $2 g$ generators. We can be more specific in the case of $J$, a principally polarized abelian variety. In this case, we have a canonical homology basis

$$
\left\{a_{1}, \ldots, a_{g}, b_{1}, \ldots, b_{g}\right\} \subset H_{1}(J, \mathbf{Z}) .
$$

That is, if we identify $H_{1}(J, \mathbf{Z})=\mathscr{L}$, then $a_{i}=\vec{\delta}_{i}$ and $b_{i}=\Omega \vec{\delta}_{i}$, where $\vec{\delta}_{i}$ is the vector in $\mathbf{Z}^{g}$ with $j$ th component $\delta_{j}^{i}$. Then we have a canonical cohomology basis denoted by

$$
\left\{x_{1}, \ldots, x_{g}, y_{1}, \ldots, y_{g}\right\} \subset H^{1}(J, \mathbf{Z}),
$$

where $x_{i}, y_{i} \in H^{1}(J, \mathbf{Z})=\operatorname{Hom}\left(H_{1}(J, \mathbf{Z}), \mathbf{Z}\right)$ are defined by

$$
\begin{array}{lll}
x_{i}\left(a_{j}\right)=\delta_{j}^{i} & \text { and } & x_{i}\left(b_{j}\right)=0, \\
y_{i}\left(a_{j}\right)=0 & \text { and } & y_{i}\left(b_{j}\right)=\delta_{j}^{i} .
\end{array}
$$

Then the cohomology ring of $J$ is the exterior algebra on these elements:

$$
H^{*}(J, \mathbf{Z})=\bigwedge\left[x_{1}, \ldots, x_{g}, y_{1}, \ldots, y_{g}\right] .
$$

The Chern classes of $\rho_{t}$ and $\xi$ are

$$
c\left(\rho_{t}\right)=1, \quad c(\xi)=1+\theta
$$

where $\theta=[\Theta]=\sum_{i=1}^{g} x_{i} \wedge y_{i} \in H^{2}(J, \mathbf{Z})$ is the class of the theta locus. This cohomology class will play an important recurring role. Note also that

$$
\theta^{n}=n ! \sum x_{i_{1}} \wedge y_{i_{1}} \wedge \cdots \wedge x_{i_{n}} \wedge y_{i_{n}},
$$

where the sum ranges over all subsets $\left\{i_{1}, \ldots, i_{n}\right\} \subset\{1, \ldots, g\}$. So it makes sense to speak of

$$
\frac{\theta^{n}}{n !} \in H^{2 n}(V, \mathbf{Z})
$$




\section{THE BUNDLES $\chi$ AND $\chi^{n}$}

Fix a marked Riemann surface $M$ of genus $g$. We will recall the definition of the Picard bundles, and we will make precise the definitions of the factors of automorphy $\chi^{n}$ and $\chi$.

We know $\Gamma(J, \xi) \rightarrow \Gamma\left(M, \rho_{r} \zeta^{g}\right)$ by restriction. We will concentrate on the maps

$$
\Gamma\left(J, \rho_{t-2 r} \xi^{2}\right) \rightarrow \Gamma\left(M, \rho_{t} \zeta^{2 g}\right) .
$$

We know from [6] that this map is surjective for each $t \in \mathbf{C}^{g}$. Also, each $\Gamma\left(J, \rho_{t-2 r} \xi^{2}\right)$ has dimension $2^{g}$ and each $\Gamma\left(M, \rho_{t} \zeta^{2 g}\right)$ has dimension $g+1$.

The Clifford bundle $\chi$. We define the factor of automorphy $\chi$ as follows. The vector of second order theta functions, $\vec{\theta}_{2}[2 r-t](w)$, viewed as a vector function of $t$, transform in the following manner [6]: for $\lambda=p+\Omega q \in \mathscr{L}$,

$$
\vec{\theta}_{2}[2 r-(t+\lambda)](w)=\rho_{w}(\lambda) \rho_{r}(\lambda) \exp \left(-\pi i^{t} q\left(t+\frac{1}{2} \Omega q\right)\right) \chi(\lambda) \vec{\theta}_{2}[2 r-t](w),
$$

where $\chi(\lambda)$ is a $2^{g} \times 2^{g}$ matrix with $\nu \mu$-components

$$
\chi(\lambda)_{\nu \mu}=\delta_{\mu+q}^{\nu} \exp \pi i^{t} p \cdot \nu .
$$

Define the factor of automorphy $\chi$ of rank $2^{g}$ by

$$
\chi(t, \lambda)=\rho_{r}(\lambda) \exp \left(-\pi i^{t} q\left(t+\frac{1}{2} \Omega q\right)\right) \chi(\lambda)
$$

so that we have

$$
\vec{\theta}_{2}[2 r-(t+\lambda)](w)=\rho_{w}(\lambda) \chi(t, \lambda) \vec{\theta}_{2}[2 r-t](w) .
$$

This says $\vec{\theta}_{2}[2 r-t](w) \in \Gamma\left(J, \rho_{w} \chi\right)$.

We call this bundle $\chi$ the Clifford bundle. This terminology is chosen because the collection of matrices $\chi(\lambda)$ form a Clifford algebra.

Note that $\chi(\lambda)$ as defined in (1) is not quite a factor of automorphy. One can check that $\chi\left(\lambda_{1}\right) \chi\left(\lambda_{2}\right)=(-1)^{{ }^{t} p_{1} \cdot q_{2}+{ }^{t} p_{2} \cdot q_{1}} \chi\left(\lambda_{2}\right) \chi\left(\lambda_{1}\right)$. Also, since $\chi(2 \lambda)_{\nu \mu}=$ $\delta_{\mu+2 q}^{\nu} \exp \pi i^{t} 2 p \cdot \nu=\delta_{\mu}^{\nu} \cdot 1$, we have that $\chi(2 \lambda)=$ Id, the identity matrix.

Recall the notation

$$
\theta_{2}\langle c \mid 2 r-t\rangle(w)={ }^{t} c \cdot \vec{\theta}_{2}[2 r-t](w),
$$

for any $c \in \mathbf{C}^{2^{8}}$. Then

$$
\begin{aligned}
\theta_{2}\langle c \mid 2 r-(t+\lambda)\rangle(w) & ={ }^{t} c \cdot \vec{\theta}_{2}[2 r-(t+\lambda)](w) \\
& ={ }^{t} c \cdot \rho_{w}(\lambda) \chi(t, \lambda) \vec{\theta}_{2}[2 r-t](w) \\
& \left.=\rho_{w}(\lambda)^{t}{ }^{t} \chi(t, \lambda) c\right) \cdot \vec{\theta}_{2}[2 r-t](w) \\
& =\rho_{w}(\lambda) \theta_{2}\left\langle^{t} \chi(t, \lambda) c \mid 2 r-t\right\rangle(w) .
\end{aligned}
$$

Hence we have

$$
\theta_{2}\left\langle\chi^{*}(t, \lambda) c \mid 2 r-(t+\lambda)\right\rangle(w)=\rho_{w}(\lambda) \theta_{2}\langle c \mid 2 r-t\rangle(w),
$$

where $\chi^{*}(t, \lambda)={ }^{t} \chi(t, \lambda)^{-1}$ is the dual factor of automorphy. 
The bundle $\chi^{*}$. For any $\lambda \in \mathscr{L}$, define $h_{\lambda}(w)=\exp 2 \pi i^{t} q \cdot w$, a function of $w \in \mathbf{C}^{g}$. This gives a nonzero section $h_{\lambda}(w) \in \Gamma\left(J, \rho_{\lambda}\right)$, and exhibits the isomorphism $\mathscr{I} \cong \rho_{\lambda}$. We obtain from this isomorphism the natural identification

$$
\Gamma\left(J, \rho_{t-2 r} \xi^{2}\right) \stackrel{\times h_{\lambda}(w)}{\cong} \Gamma\left(J, \rho_{t+\lambda-2 r} \xi^{2}\right) .
$$

This is natural in the sense that for

$$
\Gamma\left(J, \rho_{t-2 r} \xi^{2}\right) \stackrel{\times h_{\lambda_{1}}(w)}{\cong} \Gamma\left(J, \rho_{t+\lambda_{1}-2 r} \xi^{2}\right) \stackrel{\times h_{\lambda_{2}}(w)}{\cong} \Gamma\left(J, \rho_{t+\lambda_{1}+\lambda_{2}-2 r} \xi^{2}\right),
$$

we have that

$$
h_{\lambda_{1}+\lambda_{2}}(w)=h_{\lambda_{1}}(w) h_{\lambda_{2}}(w) .
$$

Hence for each $t \in \mathbf{C}^{g},\left\{\Gamma\left(J, \rho_{t+\lambda-2 r} \xi^{2}\right)\right\}_{\lambda \in \mathscr{L}}$ are all naturally identified. Then we can form a rank $2^{g}$ vector bundle over $J=\mathrm{C}^{g} / \mathscr{L}$ whose fibre over $t$ is $\Gamma\left(J, \rho_{t-2 r} \xi^{2}\right)$. Precisely, the total space is $\coprod_{t \in J} \Gamma\left(J, \rho_{t-2 r} \xi^{2}\right)$. We now show that this bundle is actually $E\left(\chi^{*}\right)$. The total space is really

$$
\coprod_{t \in \mathbf{C}^{8}} t \times \Gamma\left(J, \rho_{t-2 r} \xi^{2}\right) /(\sim),
$$

where $(t, f(w)) \sim\left(t+\lambda, h_{\lambda}(w) f(w)\right)$ for $f(w) \in \Gamma\left(J, \rho_{t-2 r} \xi^{2}\right)$. So for any $c \in \mathbf{C}^{2^{8}}$,

$$
\left(t, \theta_{2}\langle c \mid 2 r-t\rangle(w)\right) \sim\left(t+\lambda, h_{\lambda}(w) \theta_{2}\langle c \mid 2 r-t\rangle(w)\right) .
$$

The right-hand side equals $\theta_{2}\left\langle\chi^{*}(t, \lambda) c \mid 2 r-(t+\lambda)\right\rangle(w)$ by noting that $h_{\lambda}(w)$ $=\rho_{w}(\lambda)$ in (3). Using the canonical identification $\Gamma\left(J, \rho_{t-2 r} \xi^{2}\right)=\mathbf{C}^{2^{8}}$, we have that the total space is $\mathbf{C}^{g} \times \mathbf{C}^{2^{8}} /(\sim)$, where $(t, c) \sim\left(t+\lambda, \chi^{*}(t, \lambda) c\right)$. By our discussions on factors of automorphy, this means that our vector bundle has factor of automorphy $\chi^{*}$,

$$
E\left(\chi^{*}\right)=\coprod_{t \in J} \Gamma\left(J, \rho_{t-2 r} \xi^{2}\right) .
$$

The Picard bundles $\chi^{n}$ and their duals $\chi^{n *}$. We now discuss the Picard bundles $\chi^{n}$. These rank $n$ vector bundles, where $n \geq g$, are defined in [7] via the generalized theta functions, $\theta_{i}^{n}(t, z)$ for $i=1, \ldots, n$, which are functions on $\mathbf{C}^{g} \times \widetilde{M}$. The essential property is that for each $t$, as functions of $z$,

$$
\theta_{i}^{n}(t, z) \in \Gamma\left(M, \rho_{t} \zeta^{n+g-1}\right)
$$

form a basis of this $n$-dimensional vector space. And as a function of $t$,

$$
\vec{\theta}^{n}(t+\lambda, z)=\rho_{w(z)}(\lambda) \chi^{n}(t, \lambda) \vec{\theta}^{n}(t, z),
$$

where $\vec{\theta}^{n}(t, z)$ is the obvious $n$-dimensional vector. This defines $\chi^{n}$ as a factor of automorphy of rank $n$, for each $n \geq g$. Now, using the natural identifications

$$
\Gamma\left(M, \rho_{t} \zeta^{n+g-1}\right) \stackrel{\times h_{\lambda}(w(z))}{\cong} \Gamma\left(M, \rho_{t+\lambda} \zeta^{n+g-1}\right),
$$


we can form a vector bundle of rank $n$ over $J$ with total space

$$
\coprod_{t \in J} \Gamma\left(M, \rho_{t} \zeta^{n+g-1}\right) \text {. }
$$

This bundle is actually $E\left(\chi^{n *}\right)$ by applying an argument similar to the argument above for $E\left(\chi^{*}\right)$. In particular, we will concentrate on the case $n=g+1$; we have

$$
E\left(\chi^{g+1^{*}}\right)=\coprod_{t \in J} \Gamma\left(M, \rho_{t} \zeta^{2 g}\right) .
$$

Now, the map $\Gamma\left(J, \rho_{t-2 r} \xi^{2}\right) \rightarrow \Gamma\left(M, \rho_{t} \zeta^{2 g}\right)$ is given by the restriction map $\theta_{2}\langle c \mid 2 r-t\rangle(w) \mapsto \theta_{2}\langle c \mid 2 r-t\rangle(w(z))$. Then the following diagram is clearly commutative.

$$
\begin{gathered}
\Gamma\left(J, \rho_{t-2 r} \xi^{2}\right) \stackrel{\times h_{\lambda}(w)}{\cong} \Gamma\left(J, \rho_{t+\lambda-2 r} \xi^{2}\right) \\
\downarrow \\
\Gamma\left(M, \rho_{t} \zeta^{2 g}\right) \stackrel{\times h_{\lambda}(w(z))}{\cong} \Gamma\left(M, \rho_{t+\lambda} \zeta^{2 g}\right)
\end{gathered}
$$

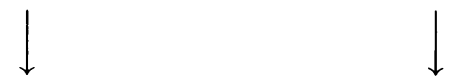

so that we get a map of vector bundles

$$
\coprod_{t \in J} \Gamma\left(J, \rho_{t-2 r} \xi^{2}\right) \rightarrow \coprod_{t \in J} \Gamma\left(M, \rho_{t} \zeta^{2 g}\right) .
$$

That is, we have

$$
\chi^{*} \rightarrow \chi^{g+1^{*}} .
$$

This is a surjective map of bundles since it is surjective on each fiber. Then equivalently, we have an injective map of vector bundles

$$
\chi^{g+1} \hookrightarrow \chi .
$$

This was proven in [6] via a different method.

Now, $\chi^{n^{*}}$ is also well known in another form. Let $N=n+g-1$, and denote $M^{[N]}=$ the $N$-fold symmetric product of $M$. It is well known that there is a one-to-one correspondence

$$
\begin{aligned}
\mathbf{P} \Gamma\left(M, \rho_{t} \zeta^{N}\right) & \leftrightarrow\left\{\left(z_{1}, \ldots, z_{N}\right) \in M^{[N]}: t=w\left(z_{1}\right)+\cdots+w\left(z_{N}\right) \in J\right\}, \\
f(z) & \leftrightarrow \operatorname{Div}(f) .
\end{aligned}
$$

Thus

$$
\begin{array}{r}
\mathbf{P} E\left(\chi^{n *}\right)=M^{[N]} \\
\downarrow \\
J,
\end{array}
$$

where $M^{[N]} \rightarrow J$ is a classically studied projective bundle. In fact $E\left(\chi^{n *}\right)$ is the canonical lift of this projective bundle to a vector bundle that Mattuck and Kempf refers to as the Picard bundle. We will for some consistency reason instead refer to $\chi^{n}$ as the Picard bundle. 
It is worth taking a little time to explain why our bundle $\chi^{n *}$ is the same vector bundle Mattuck constructed in [17] since we will be using his calculation of its Chern classes. In general, given a projective bundle, the isomorphism class of a lift of this projective bundle is determined by a codimension one divisor on the total space of the projective bundle which happens to be the Chern class of the resulting universal line bundle. The divisor class specifying the lift that Mattuck constructed is what he denoted as $X\left[p_{0}\right]$,

$$
X\left[p_{0}\right]=\left\{p_{0}+z_{1}+\cdots+z_{N-1} \in M^{[N]}: z_{i} \in M \text { arbitrary }\right\} \subset M^{[N]} .
$$

where $p_{0}$ is the image of the base point $z_{0}$ in $M$. The universal line bundle on $\mathbf{P} E\left(\chi^{n *}\right)$ is the dual to the line bundle whose fiber over $[f] \in \mathbf{P} \Gamma\left(M, \rho_{t} \zeta^{N}\right)$ is the line spanned by $f \in \Gamma\left(M, \rho_{t} \zeta^{N}\right)$. We can give a section of the universal line bundle as follows. For any $[f] \in \mathbf{P} \Gamma\left(M, \rho_{t} \zeta^{N}\right)$, define $s([f]) \in$ $\operatorname{Hom}(\operatorname{Span}\{f\}, \mathbf{C})$ by

$$
s([f]): f \mapsto f\left(z_{0}\right) .
$$

We have to check that this is well defined. This is clearly independent of the choice of the representative $f \in[f]$. More importantly, we have identified $\Gamma\left(M, \rho_{t} \zeta^{N}\right) \stackrel{\times h_{\lambda}(w(z))}{\rightarrow} \Gamma\left(M, \rho_{t+\lambda} \zeta^{N}\right)$, so that $f(z)$ corresponds to $h_{\lambda}(w(z)) f(z)$. Then all we need is that $f\left(z_{0}\right)=h_{\lambda}\left(w\left(z_{0}\right)\right) f\left(z_{0}\right)$, which is indeed true since $w\left(z_{0}\right)=0$ and $h_{\lambda}(0)=1$. The zeroes of this section $s$ is precisely where $f\left(z_{0}\right)=0$. This happens precisely when the divisor of $f$, viewed as a divisor on $M$, contains $p_{0}$. We have

$$
\operatorname{Zero}(s)=\left\{f: \operatorname{Div}(f)=p_{0}+z_{1}+\cdots+z_{N-1}\right\} .
$$

This gives the Chern class of the universal line bundle. By the identification (3) of $\mathbf{P} E\left(\chi^{n *}\right)$ with $M^{[N]}$, we see that $\operatorname{Zero}(s)=X\left[p_{0}\right]$ exactly. So indeed, the vector bundle $\chi^{n *}$ is precisely the one Mattuck dealt with in [17].

Mattuck computed the Chern classes of $\chi^{n *}$ to be

$$
c_{i}\left(\chi^{n *}\right)=(-1)^{i}\left[W_{i}\right] \in H^{2 i}(J, \mathbf{Z}),
$$

where $W_{i}=$ the image of $M^{[i]}$ in $J$. We know $\left[W_{i}\right]=\theta^{i} / i$ ! . Hence

$$
c\left(\chi^{n *}\right)=1-\theta+\frac{\theta^{2}}{2 !}-\cdots+(-1)^{g} \frac{\theta^{g}}{g !}
$$

which we can write simply as

$$
c\left(\chi^{n *}\right)=e^{-\theta} \in H^{*}(J, \mathbf{Z}) .
$$

Therefore the Chern class of the dual vector bundle is

$$
c\left(\chi^{n}\right)=e^{\theta}=1+\theta+\frac{\theta^{2}}{2 !}+\cdots+\frac{\theta^{g}}{g !} .
$$

Note that $c\left(\chi^{n}\right)$ and $c\left(\chi^{n *}\right)$ are independent of $n$. 


\section{The BUNDles $\chi$ AND $\chi^{n}$ AND CERTAIN PUllbacks}

In order to study the question of how many second order theta functions $\theta_{2}\langle c \mid 2 r-t\rangle(w(z))$ it takes to span $\Gamma^{\prime}\left(M, \rho_{t} \zeta^{2 g}\right)$ for all $t$, we need to study the pullbacks of the Clifford and Picard bundles under the doubling map $m: J \stackrel{\times 2}{\rightarrow}$ $J$. The reason, as we will see later, is that the span of a second order theta function will not form a line subbundle of the Clifford bundle unless we we pull back by this map.

For any bundle $\eta$ on $J$, denote its pullback by $\tilde{\eta}=m^{*} \eta$. The bundle $\tilde{\eta}$ has fiber at $t \in J$ given by

$$
E(\widetilde{\eta})_{t}=E(\eta)_{2 t} .
$$

In terms of factors of automorphy, we have that

$$
\widetilde{\eta}(t, \lambda)=\eta(2 t, 2 \lambda) .
$$

Note that $m$ is a finite covering map of degree $2^{2 g}$.

The induced cohomology map. It is easy to see that the map $m$ takes each canonical 1-cycle to twice itself, so

$$
m_{*} a_{i}=2 a_{i} \text { and } m_{*} b_{i}=2 b_{i} .
$$

This implies that the induced map on the first homology groups

$$
m_{*}: H_{1}(J, \mathbf{Z}) \stackrel{\times 2}{\rightarrow} H_{1}(J, \mathbf{Z})
$$

is multiplication by 2 . Since $H^{1}(J, \mathbf{Z})=\operatorname{Hom}\left(H_{1}(J, \mathbf{Z}), \mathbf{Z}\right)$, we have that

$$
m^{*}: H^{1}(J, \mathbf{Z}) \stackrel{\times 2}{\rightarrow} H^{1}(J, \mathbf{Z})
$$

is also multiplication by 2 , so

$$
m^{*} x_{i}=2 x_{i} \text { and } m^{*} y_{i}=2 y_{i} .
$$

In particular, we have that

$$
m^{*} \theta=\sum_{i=1}^{g} 2 x_{i} \wedge 2 y_{i}=4 \sum_{i=1}^{g} x_{i} \wedge y_{i}=4 \theta .
$$

Note that $m^{*}: H^{*}(J, \mathbf{Z}) \rightarrow H^{*}(J, \mathbf{Z})$ is an injective ring homomorphism.

The bundle $\tilde{\chi}$. Now consider the vector bundle $\chi$. Its pullback via $m$ has factor of automorphy

$$
\begin{aligned}
\tilde{\chi}(t, \lambda) & =\chi(2 t, 2 \lambda) \\
& =\rho_{r}(2 \lambda) \exp \left(-\pi i^{t}(2 q)\left(2 t+\frac{1}{2} \Omega 2 q\right)\right) \chi(2 \lambda) \\
& =\rho_{2 r}(\lambda) \exp 2\left(-2 \pi i^{t} q\left(t+\frac{1}{2} \Omega q\right)\right) \mathrm{Id} \\
& =\rho_{2 r}(\lambda) \xi(t, \lambda)^{2} \mathrm{Id},
\end{aligned}
$$


where Id is the $2^{g} \times 2^{g}$ identity matrix. Thus $\tilde{\chi}$ is a scalar bundle with

$$
\tilde{\chi}=\bigoplus_{j=1}^{2^{g}} \rho_{2 r} \xi^{2}
$$

as bundles on $J$. Then the Chern class of this bundle is

$$
c(\tilde{\chi})=\prod_{j=1}^{2^{g}} c\left(\rho_{2 r} \xi^{2}\right)=(1+2 \theta)^{2^{g}},
$$

by using the formula for the Chern class of a direct sum and the fact that $c\left(\rho_{2 r} \xi^{2}\right)=1+2 \theta$. By using the formula for the Chern class of a dual, we have that $c\left(\widetilde{\chi^{*}}\right)=(1-2 \theta)^{2^{8}}$. This proves

Proposition 1. We have isomorphisms $\tilde{\chi}=\rho_{2 r} \xi^{2} \mathscr{I}_{2^{g}}$ and $\widetilde{\chi^{*}}=\rho_{-2 r} \xi^{-2} \mathscr{I}_{2^{g}}$, and so their Chern classes are

$$
c(\tilde{\chi})=(1+2 \theta)^{2^{8}}, \quad c\left(\widetilde{\chi^{*}}\right)=(1-2 \theta)^{2^{8}} .
$$

Next we wish to calculate $c(\chi)$. We need the following proposition.

Proposition 2. It makes sense to write $\left(1+\frac{1}{2} \theta\right)^{2^{8}} \in H^{*}(J, \mathbf{Z})$.

Proof. We have the expansion

$$
\left(1+\frac{1}{2} \theta\right)^{2^{g}}=1+\frac{1}{2} 2^{g} \theta+\frac{1}{2^{2}}\left(\begin{array}{c}
2^{g} \\
2
\end{array}\right) \theta^{2}+\cdots+\frac{1}{2^{g}}\left(\begin{array}{c}
2^{g} \\
g
\end{array}\right) \theta^{g}
$$

Instead, we can write

$$
\left(1+\frac{1}{2} \theta\right)^{2^{g}}=1+\frac{1}{2} 2^{g} \theta+\frac{2 !}{2^{2}}\left(\begin{array}{c}
2^{g} \\
2
\end{array}\right) \frac{\theta^{2}}{2 !}+\cdots+\frac{g !}{2^{g}}\left(\begin{array}{c}
2^{g} \\
g
\end{array}\right) \frac{\theta^{g}}{g !} .
$$

We know that $\theta^{k} / k ! \in H^{2 k}(J, \mathbf{Z})$. But the coefficient of $\theta^{k} / k$ ! is just

$$
\begin{aligned}
\frac{k !}{2^{k}}\left(\begin{array}{c}
2^{k} \\
k
\end{array}\right) & =\frac{k !}{2^{k}} \frac{2^{g}\left(2^{g}-1\right) \cdots\left(2^{g}-k+1\right)}{1 \cdot 2 \cdots k} \\
& =\frac{2^{g}\left(2^{g}-1\right) \cdots\left(2^{g}-k+1\right)}{2^{k}},
\end{aligned}
$$

which is clearly an integer for $1 \leq k \leq g$. Thus indeed $\left(1+\frac{1}{2} \theta\right)^{2^{8}} \in H^{*}(J, \mathbf{Z})$ as claimed.

Now, since $m^{*} \theta=4 \theta$, we have that

$$
m^{*}\left(\left(1+\frac{1}{2} \theta\right)^{2^{8}}\right)=(1+2 \theta)^{2^{8}}=c(\tilde{\chi}) .
$$

But we know that $m^{*} c(\chi)=c(\tilde{\chi})$, so $m^{*}(c(\chi))=m^{*}\left(\left(1+\frac{1}{2} \theta\right)^{2^{8}}\right)$. Since $m^{*}$ is an injective homomorphism, we must have that $c(\chi)=\left(1+\frac{1}{2} \theta\right)^{2^{8}}$. This proves the following. 
Proposition 3. The Chern classes of $\chi$ and $\chi^{*}$ are

$$
c(\chi)=\left(1+\frac{1}{2} \theta\right)^{2^{8}}, \quad c\left(\chi^{*}\right)=\left(1-\frac{1}{2} \theta\right)^{2^{8}} .
$$

The bundle $\widetilde{\chi^{*}}$. Recall that $E\left(\chi^{*}\right)$ was constructed as $\amalg_{t \in J} \Gamma\left(\rho_{t-2 r} \xi^{2}\right)$ with the natural identifications $\Gamma\left(\rho_{t-2 r} \xi^{2}\right)=\Gamma\left(\rho_{t+\lambda-2 r} \xi^{2}\right)$ for $\lambda \in \mathscr{L}$. It is clear that

$$
E\left(\widetilde{\chi^{*}}\right)=\coprod_{t \in J} \Gamma\left(\rho_{2 t-2 r} \xi^{2}\right)
$$

with the identifications $\Gamma\left(\rho_{2 t-2 r} \xi^{2}\right)=\Gamma\left(\rho_{2 t+2 \lambda-2 r} \xi^{2}\right)$ for $\lambda \in \mathscr{L}$. Now for a fixed nonzero $c \in \mathbf{C}^{2^{8}}$, consider the one-dimensional subspace described by

$$
\operatorname{Span}\left\{\theta_{2}\langle c \mid 2 r-2 t\rangle(w)\right\} \subset \Gamma\left(\rho_{2 t-2 r} \xi^{2}\right)
$$

as $t$ varies. Let $\lambda=p+\Omega q \in \mathscr{L}$. We have from equation (2) that

$$
\rho_{w}(2 \lambda) \theta_{2}\langle c \mid 2 r-2 t\rangle(w)=\theta_{2}\left\langle\chi^{*}(2 t, 2 \lambda) c \mid 2 r-2(t+\lambda)\right\rangle(w) .
$$

But from previous calculations, we know that

$$
\chi^{*}(2 t, 2 \lambda)=\rho_{-2 r}(\lambda) \xi(t, \lambda)^{-2} \mathrm{Id} .
$$

Hence we have

(4) $\rho_{-2 r}(\lambda) \xi(t, \lambda)^{-2} \theta_{2}\langle c \mid 2 r-2(t+\lambda)\rangle(w)=h_{2 \lambda}(w) \theta_{2}\langle c \mid 2 r-2 t\rangle(w)$.

The natural identification of

$$
\Gamma\left(\rho_{2 t-2 r} \xi^{2}\right)=\Gamma\left(\rho_{2 t+2 \lambda-2 r} \xi^{2}\right)
$$

identifies $\theta_{2}\langle c \mid 2 r-2 t\rangle(w)$ with $h_{2 \lambda}(w) \theta_{2}\langle c \mid 2 r-2 t\rangle(w)$. Thus under this identification, $\theta_{2}\langle c \mid 2 r-2(t+\lambda)\rangle(w)$ and $\theta_{2}\langle c \mid 2 r-2 t\rangle(w)$ differ only by a scalar factor of $\rho_{-2 r}(\lambda) \xi(t, \lambda)^{-2}$. In particular, this implies that

$$
\operatorname{Span}\left\{\theta_{2}\langle c \mid 2 r-2 t\rangle(w)\right\}=\operatorname{Span}\left\{\theta_{2}\langle c \mid 2 r-2(t+\lambda)\rangle(w)\right\},
$$

and hence we can say

$$
\coprod_{t \in J} \operatorname{Span}\left\{\theta_{2}\langle c \mid 2 r-2 t\rangle(w)\right\} \subset \coprod_{t \in J} \Gamma\left(\rho_{2 t-2 r} \xi^{2}\right) .
$$

This defines a new line subbundle of $\widetilde{\chi^{*}}$ which we shall denote by $\mu_{c}^{*}$. This proves

Proposition 4. For each nonzero vector $c \in \mathbf{C}^{2^{8}}$, we can define a line subbundle $\mu_{c}^{*} \hookrightarrow \widetilde{\chi^{*}} b y$

$$
E\left(\mu_{c}^{*}\right)=\coprod_{t \in J} \operatorname{Span}\left\{\theta_{2}\langle c \mid 2 r-2 t\rangle(w)\right\} \subset E\left(\widetilde{\chi^{*}}\right) .
$$

These line bundles $\mu_{c}^{*}$ are useful in studying questions about the behavior of second order theta functions when restricted to the curve $M$. In this case the 
relevant map is $\mu_{c}^{*} \rightarrow \chi^{\widetilde{g+1^{*}}}$, which comes from the composition $\mu_{c}^{*} \hookrightarrow \widetilde{\chi^{*}} \rightarrow$ $\widetilde{\chi^{g+1^{*}}}$. Here, by reasoning similar to the discussion on $\widetilde{\chi^{*}}$, we have that

$$
E\left(\chi^{\widetilde{g+1^{*}}}\right)=\coprod_{t \in J} \Gamma\left(M, \rho_{2 t} \xi^{2 g}\right),
$$

and we have

$$
c\left(\chi^{\widetilde{g+1}}\right)=e^{-4 \theta}
$$

For instance, suppose we are interested in the interplay of some basic second order theta functions $\left\{\theta_{2}\left\langle c_{i} \mid 2 r-t\right\rangle(w)\right\}_{i=1}^{n}$ when restricted to the curve $M$ as $t$ varies. We can consider $\theta_{2}\left\langle c_{i} \mid 2 r-2 t\right\rangle(w)$ without changing the question. We would look at the map

$$
\mu_{c_{1}}^{*} \oplus \cdots \oplus \mu_{c_{n}}^{*} \rightarrow \widetilde{\chi^{g+1}}
$$

of vector bundles over $J$. It is clear that the image of this map on each fiber is

$$
\operatorname{Span}\left\{\theta_{2}\left\langle c_{i} \mid 2 r-2 t\right\rangle(w(z))\right\}_{i=1}^{n} \subset \Gamma\left(M, \rho_{2 t} \xi^{2 g}\right),
$$

so we have the following remark.

Remark 1 . The restricted second order theta functions

$$
\left\{\theta_{2}\left\langle c_{i} \mid 2 r-2 t\right\rangle(w(z))\right\}_{i=1, \ldots, n}
$$

are linearly independent for a fixed $t$ if and only if the map (5) is injective at $t$. And $\left\{\theta_{2}\left\langle c_{i} \mid 2 r-2 t\right\rangle(w(z))\right\}$ span $\Gamma\left(M, \rho_{2 t} \xi^{2 g}\right)$ for a fixed $t$ if and only if the map (5) is surjective at $t$. Furthermore, $\left\{\theta_{2}\left\langle c_{i} \mid 2 r-2 t\right\rangle(w(z))\right\}$ are linearly independent for all $t \in \mathbf{C}^{g}$ if and only (5) is an injective map of vector bundles, and $\left\{\theta_{2}\left\langle c_{i} \mid 2 r-2 t\right\rangle(w(z))\right\}$ span $\Gamma\left(M, \rho_{2 t} \xi^{2 g}\right)$ for all $t \in \mathbf{C}^{g}$ if and only if $(5)$ is a surjective map of vector bundles.

Proposition 5. For any nonzero $c \in \mathbf{C}^{2^{8}}$, we have an isomorphism $\mu_{c}^{*} \cong \rho_{-2 r} \xi^{-2}$, and so the Chern class of $\mu_{c}^{*}$ is $c\left(\mu_{c}^{*}\right)=1-2 \theta$.

Proof. Identify

$$
\operatorname{Span}\left\{\theta_{2}\langle c \mid 2 r-2 t\rangle(w)\right\}=t \times \mathbf{C}
$$

by identifying $\theta_{2}\langle c \mid 2 r-2 t\rangle(w) \leftrightarrow t \times 1$. Then

$$
E\left(\mu_{c}^{*}\right)=\coprod_{t \in \mathbf{C}^{8}} \operatorname{Span}\left\{\theta_{2}\langle c \mid 2 r-2 t\rangle(w)\right\} /(\sim)=\coprod_{t \in \mathbf{C}^{8}} t \times \mathbf{C} /(\sim),
$$

where we want $\left(t, \alpha \theta_{2}\langle c \mid 2 r-2 t\rangle(w)\right) \sim\left(t+\lambda, h_{2 \lambda}(w) \alpha \theta_{2}\langle c \mid 2 r-2 t\rangle(w)\right)$ for $t \in \mathbf{C}^{g}, \alpha \in \mathbf{C}$, and $\lambda \in \widetilde{\mathscr{L}}$. By equation (4), this is actually

$$
\left(t, \alpha \theta_{2}\langle c \mid 2 r-2 t\rangle(w)\right) \sim\left(t+\lambda, \rho_{-2 r}(\lambda) \xi(t, \lambda)^{-2} \alpha \theta_{2}\langle c \mid 2 r-2 t\rangle(w)\right) .
$$

By our identifications, this becomes $(t, \alpha) \sim\left(t+\lambda, \rho_{-2 r}(\lambda) \xi(t, \lambda)^{-2} \alpha\right)$. Hence the factor of automorphy of $\mu_{c}^{*}$ is $\mu_{c}^{*}(t, \lambda)=\rho_{-2 r}(\lambda) \xi(t, \lambda)^{-2}$, which was to be proven. 
Remark 2. We see that the factor of automorphy $\mu_{c}^{*}$ is independent of $c \in \mathbf{C}^{2^{8}}$. That is, all the different $\mu_{c}^{*}$ are isomorphic as vector bundles.

Remark 3. For any choice of a basis $c_{1}, \ldots, c_{2^{8}}$ of $\mathbf{C}^{2^{8}}$, the map $\mu_{c_{1}}^{*} \oplus \cdots \oplus$ $\mu_{c_{2} g}^{*} \rightarrow \widetilde{\chi^{*}}$ is an isomorphism.

The notation $\mu_{c}^{*}$ was chosen so as to be consistent and suggestive. The dual map of the injection $\mu_{c}^{*} \hookrightarrow \chi^{*}$ is the surjection $\chi \rightarrow \mu_{c}$, where $\mu_{c}$ is the dual line bundle of $\mu_{c}^{*}$. This dual bundle has Chern class $c\left(\mu_{c}\right)=1+2 \theta$.

\section{THE OBJECTIVE}

We now deal with the problem of how many basic second order theta functions of the form $\theta_{2}\langle c \mid 2 r-t\rangle(w(z))$ does it take to insure that they span $\Gamma\left(\rho_{t} \zeta^{2 g}\right)$ for all $t$.

It would be nice but not very interesting if the job could be done with just $g+1$ of them. At any fixed $t_{0} \in \mathbf{C}^{g}$, we can certainly find $g+1$ vectors $c_{1}, \ldots, c_{g+1} \in \mathbf{C}^{2^{g}}$ such that $\left\{\theta_{2}\left\langle c_{i} \mid 2 r-t_{0}\right\rangle(w(z))\right\}_{i=1}^{g+1}$ is a basis for $\Gamma\left(\rho_{t_{0}} \zeta^{2 g}\right)$. However, this choice of $g+1$ second order theta functions might not be a basis of $\Gamma\left(\rho_{t} \zeta^{2 g}\right)$ for some other values of $t$. In fact we have the following.

Proposition 6. For any choice of $c_{1}, \ldots, c_{g+1} \in \mathbf{C}^{2^{8}}$, let

$$
X=\left\{t \in \mathbf{C}^{g}: \operatorname{Span}\left\{\theta_{2}\left\langle c_{i} \mid 2 r-2 t\right\rangle(w(z))\right\}_{i=1}^{g+1} \neq \Gamma\left(\rho_{2 t} \zeta^{2 g}\right)\right\}
$$

be the subvariety where these restricted theta functions fail to form a basis. Then viewing $X \subset J$, we must have either $X=J$ or $[X]=(2 g-2) \theta \in H^{2}(J, Z)$. In particular, $X$ cannot be empty for $g>1$.

Proof. Let $\mu^{*}=\mu_{c_{1}}^{*} \oplus \cdots \oplus \mu_{c_{g+1}}^{*}$. Then $X$ is precisely the degeneracy locus of the map $\mu^{*} \rightarrow \widetilde{\chi^{g+1^{*}}}$. We will apply Porteous' formula, as stated in [2] or [4]. Each irreducible component of $X=X_{g}\left(\mu^{*} \rightarrow \widetilde{\chi^{g+1^{*}}}\right)$ has dimension at least $\operatorname{dim} J-(g+1-g)(g+1-g)=\operatorname{dim} J-1=g-1$. If $X$ is either empty or $\operatorname{dim} X=g-1$, then $[X]=\Delta_{1,1}\left(c\left(\chi^{g+1^{*}}\right) / c\left(\mu^{*}\right)\right)$. We have

$$
\begin{aligned}
c\left(\chi^{g+1^{*}}\right) / c\left(\mu^{*}\right) & =e^{-4 \theta} /(1-2 \theta)^{g+1} \\
& =e^{-4 \theta}\left(1+2 \theta+4 \theta+\cdots+2^{g} \theta^{g}\right)^{g+1} \\
& =(1-4 \theta+\cdots)(1+(2 g+2) \theta+\cdots) \\
& =1+(2 g-2) \theta+\cdots,
\end{aligned}
$$

so that $[X]=(2 g-2) \theta$ in this case. Otherwise, $X$ has a component of dimension greater than $g-1$, which must necessary be of dimension $g$. Since $J$ is an irreducible space, this forces $X=J$. This completes the proof. 
Remark 4. For genus $g=1$, we have $2^{g}=2=g+1$ so that the Clifford bundle $\chi$ and the Picard bundle $\chi^{g+1}$ are the same bundle. In this case, any two linearly independent $c_{1}, c_{2}$ would produce two $\theta_{2}\left\langle c_{i} \mid 2 r-t\right\rangle(w(z))$ that span $\Gamma\left(M, \rho_{t} \zeta^{2}\right)$ for all $t$. Clearly this must be the case since $M \cong J$ in genus 1 .

Remark 5. For genus $g=2$, we have $2^{g}=4$ and $g+1=3$. So we know we can find 4 vectors $c_{1}, c_{2}, c_{3}, c_{4}$ such that $\left\{\theta_{2}\left\langle c_{i} \mid 2 r-t\right\rangle(w(z))\right\}$ span $\Gamma\left(M, \rho_{t} \zeta^{4}\right)$ for all $t$. This is in fact the minimum number since by Proposition 6 we know it cannot be done with $g+1=3$.

In fact for genus $g>1$, these restricted second order theta functions interplay much more. The next theorem says that any choice of $t w o$ such restricted second order theta functions cannot be linearly independent for all $t$. Therefore, a priori, we see that $g+1$ of them cannot hope to be linearly independent for all $t$.

Theorem 1. Assume genus $g>1$. For any two vectors, $c_{1}, c_{2} \in \mathbf{C}^{2^{8}}$, there exists some $t_{0} \in \mathbf{C}^{g}$ such that

$$
\theta_{2}\left\langle c_{1} \mid 2 r-t_{0}\right\rangle(w(z)) \text { and } \theta_{2}\left\langle c_{2} \mid 2 r-t_{0}\right\rangle(w(z))
$$

are linearly dependent as functions of $z \in \widetilde{M}$.

Proof. Assume $c_{1}, c_{2}$ are linearly independent, for otherwise there is nothing to prove. The two corresponding theta functions describe a subbundle $\mu_{c_{1}}^{*} \oplus \mu_{c_{2}}^{*} \hookrightarrow$ $\widetilde{\chi^{*}}$, which for simplicity we will denote by $\mu^{*}$. The restriction of the theta functions to $\widetilde{M}$ yields the map $\mu^{*} \rightarrow \widetilde{\chi^{g+1^{*}}}$. Suppose by contradiction that for all $t_{0}, \theta_{2}\left\langle c_{i} \mid 2 r-t_{0}\right\rangle(w(z)), i=1,2$, remained linearly independent. By remark 1 , this is equivalent to saying that the above map is injective. Then we have an exact sequence

$$
0 \rightarrow \mu^{*} \rightarrow \chi^{\widetilde{g+1^{*}}} \rightarrow Q \rightarrow 0
$$

of bundles over $J$. Since $Q$ has rank $g+1-2=g-1$, its $g$ th Chern class must be zero. We know that $c\left(\mu^{*}\right)=(1-2 \theta)^{2}$ and $c\left(\chi^{g+1^{*}}\right)=e^{-4 \theta}$, so that

$$
\begin{aligned}
c(Q) & =c\left(\mu^{*}\right) / c\left(\widetilde{\chi^{g+1}}\right) \\
& =e^{-4 \theta} /(1-2 \theta)^{2} \\
& =e^{-4 \theta}\left(1+2 \theta+4 \theta^{2}+\cdots+2^{g} \theta^{g}\right)^{2} \\
& =\left(\sum_{j=0}^{g} \frac{1}{j !}(-4 \theta)^{j}\right)\left(\sum_{i=0}^{g}(i+1)(2 \theta)^{i}\right) .
\end{aligned}
$$


So the $g$ th Chern class is

(6)

$$
\begin{aligned}
c_{g}(Q) & =\sum_{k=0}^{g}\left[\frac{1}{k !}(-4 \theta)^{k}\right]\left[(g-k+1)(2 \theta)^{g-k}\right] \\
& =\sum_{k=0}^{g} \frac{g-k+1}{k !}(-2)^{k}(2 \theta)^{g} \\
& =\left(2 \theta^{g}\right)\left[\sum_{k=0}^{g}(-2)^{k} \frac{g+1}{k !}-\sum_{k=0}^{g}(-2)^{k} \frac{k}{k !}\right] \\
& =\left(2 \theta^{g}\right)\left[\frac{(g+1)(-2)^{g}}{g !}+\sum_{k=0}^{g-1}(-2)^{k} \frac{g+1}{k !}+2 \sum_{k=1}^{g}(-2)^{k-1} \frac{1}{(k-1) !}\right] \\
& =\left(2 \theta^{g}\right)\left[\frac{(g+1)(-2)^{g}}{g !}+(g+1) \sum_{k=0}^{g-1} \frac{(-2)^{k}}{k !}+2 \sum_{k=0}^{g-1} \frac{(-2)^{k}}{k !}\right] \\
& =\left(2 \theta^{g}\right)\left[\frac{(g+1)(-2)^{g}}{g !}+(g+3) \sum_{k=0}^{g-1} \frac{(-2)^{k}}{k !}\right] .
\end{aligned}
$$

But $c_{g}(Q)=0$. So we must have

$$
0=\frac{(g+1)(-2)^{g}}{g !}+(g+3) \sum_{k=0}^{g-1} \frac{(-2)^{k}}{k !} .
$$

Then necessarily,

$$
\frac{2^{g}(g+1)}{g !}=(g+3)\left|\sum_{k=0}^{g-1} \frac{(-2)^{k}}{k !}\right| .
$$

Now, the series $\sum_{k=0}^{\infty} \frac{(-2)^{k}}{k !}$ converges to $e^{-2}$. The fact that it is an alternating series whose $n$th term decreases in absolute value for $n>2$ implies that

$$
\left|e^{-2}-\sum_{k=0}^{n} \frac{(-2)^{k}}{k !}\right| \leq \frac{2^{n+1}}{(n+1) !} \quad \text { for } n>2 \text {. }
$$

Then by the triangle inequality,

$$
\left|e^{-2}\right|-\left|\sum_{k=0}^{n} \frac{(-2)^{k}}{k !}\right| \leq \frac{2^{n+1}}{(n+1) !},
$$

and so

$$
e^{-2}-\frac{2^{n+1}}{(n+1) !} \leq\left|\sum_{k=0}^{n} \frac{(-2)^{k}}{k !}\right| \quad \text { for } n>2 .
$$

So in the case $g>3$, by taking $n=g-1$, we have

$$
\left|\sum_{k=0}^{g-1} \frac{(-2)^{k}}{k !}\right| \geq e^{-2}-\frac{2^{g}}{g !} \text {. }
$$


Continuing from equation (7) and then applying this inequality, we have

$$
\begin{aligned}
2^{g}(g+1) & =g !(g+3)\left|\sum_{k=0}^{g-1} \frac{(-2)^{k}}{k !}\right| \\
& \geq g !(g+3)\left(e^{-2}-\frac{2^{g}}{g !}\right) \\
& \geq g !(g+3) e^{-2}-2^{g}(g+3) .
\end{aligned}
$$

Then

$$
2^{g}(2 g+4) \geq g !(g+3) e^{-2},
$$

and so

$$
\frac{2 g+4}{g+3} e^{2} \geq \frac{g !}{2^{g}}
$$

Using $\frac{2 g+4}{g+3}<2$ and $e<3$, we have

$$
\frac{g !}{2^{g}}<18 \text {. }
$$

But this is impossible for $g \geq 7$ since $\frac{n !}{2^{n}}$ is an increasing sequence for $n>2$, and already for $n=7, \frac{7 !}{2^{7}}=7 \cdot 720 / 128>7 \cdot 5>18$. To complete the proof by contradiction, we need to show that the relation $c_{g}(Q)=0$ is impossible also for $g=2,3,4,5,6$. But from (6), we have

$$
c_{g}(Q)=\sum_{k=0}^{g} \frac{g-k+1}{k !}(-2)^{k}(2 \theta)^{g}=\sum_{k=0}^{g} \frac{g !(g-k+1)}{k !}(-2)^{k} \cdot \frac{(2 \theta)^{g}}{g !},
$$

which by arithmetic yields the following:

$$
\begin{array}{ll}
g=2: & c_{2}(Q)=2 \cdot \frac{(2 \theta)^{2}}{2 !}, \\
g=3: & c_{3}(Q)=4 \cdot \frac{(2 \theta)^{3}}{3 !}, \\
g=4: & c_{4}(Q)=24 \cdot \frac{(2 \theta)^{4}}{4 !}, \\
g=5: & c_{5}(Q)=128 \cdot \frac{(2 \theta)^{5}}{5 !}, \\
g=6: & c_{6}(Q)=880 \cdot \frac{(2 \theta)^{6}}{6 !} .
\end{array}
$$

This completes the proof.

\section{MAIN THEOREM}

The question of exactly how many restricted second order theta functions $\theta_{2}\langle c \mid 2 r-t\rangle(w(z))$ it takes to span $\Gamma\left(\rho_{t} \zeta^{2 g}\right)$ for all $t \in \mathbf{C}^{g}$ was completely answered in Remarks 4 and 5 for genuses $g=1$ and $g=2$. We now deal with the problem for $g>2$. For this purpose we study the map $\Gamma\left(J, \rho_{t-2 r} \xi^{2}\right) \rightarrow$ $\Gamma\left(M, \rho_{t} \zeta^{2 g}\right)$ more closely. 
For each $t \in \mathbf{C}^{g}$, let $A(t) \subset \mathbf{C}^{2^{g}}$ be the kernel of

$$
\Gamma\left(J, \rho_{t-2 r} \xi^{2}\right) \rightarrow \Gamma\left(M, \rho_{t} \zeta^{2 g}\right) .
$$

That is, by using the usual identification of $\Gamma\left(J, \rho_{t-2 r} \xi^{2}\right)$ with $\mathbf{C}^{2^{8}}$, we have

$$
A(t)=\left\{c \in \mathbf{C}^{2^{8}}: \theta_{2}\langle c \mid 2 r-t\rangle(w(z)) \equiv 0 \text { in } z\right\} .
$$

Since the above map is surjective, the dimension of each $A(t)$ is $2^{g}-(g+1)$. Clearly, the collection $\{A(t)\}$ forms an analytic family of linear subspaces of $\mathbf{C}^{2^{8}}$ indexed by $t \in \mathbf{C}^{g}$. Since

$$
\theta_{2}\langle c \mid 2 r-t\rangle(w(z))=\theta_{2}\left\langle\chi^{*}(t, \lambda) c \mid 2 r-(t+\lambda)\right\rangle(w(z)),
$$

it is clear from the definition of $A(t)$ that

$$
c \in A(t) \text { if and only if } \chi^{*}(t, \lambda) c \in A(t+\lambda) .
$$

Another way to consider these $A(t)$ is the following. Let $\mathscr{K}$ be the vector bundle which is the kernel bundle of the surjection $\chi^{*} \rightarrow \chi^{g+1^{*}}$, so

$$
0 \rightarrow \mathscr{K} \rightarrow \chi^{*} \rightarrow \chi^{g+1^{*}} \rightarrow 0 .
$$

Then the total space of $\mathscr{K}$, viewed as a subset of $E\left(\chi^{*}\right)$, is exactly

$$
E(\mathscr{K})=\coprod_{t \in \mathbf{C}^{g}} A(t) /(\sim),
$$

where the equivalence $(\sim)$ is that of

$$
E\left(\chi^{*}\right)=\mathbf{C}^{g} \times \mathbf{C}^{2^{8}} /(\sim),
$$

namely, $(t, c) \sim\left(t+\lambda, \chi^{*}(t, \lambda) c\right)$. From this viewpoint, it is also easy to deduce (8).

We are interested in the "orthogonal complement" $A(t)^{\perp}$ which is taken with respect to the symmetric inner product on $\mathbf{C}^{2^{8}}$. To be precise, we have

$$
A(t)^{\perp}=\left\{a \in \mathbf{C}^{2^{8}}:{ }^{t} a \cdot c=0 \text { for all } c \in A(t)\right\},
$$

so $A(t)^{\perp}$ is a $g+1$-dimensional subspace of $\mathbf{C}^{2^{8}}$.

Note that when we say "orthogonal", it is with respect to this symmetric bilinear form and not the Hermitian one; the reason is that we wish to keep all relations holomorphic.

Proposition 7. For any $\lambda \in \mathscr{L}$, we have

$$
c \in A(t)^{\perp} \quad \text { if and only if } \chi(t, \lambda) c \in A(t+\lambda)^{\perp} .
$$


Proof. We have the following equivalences:

$$
\begin{aligned}
c \in A(t) & \Leftrightarrow{ }^{t} c \cdot a=0 \quad(\text { for all } a \in A(t)) \\
& \Leftrightarrow{ }^{t} c \cdot a=0 \quad\left(\text { for all } \chi^{*}(t, \lambda) a \in A(t+\lambda)\right) \\
& \Leftrightarrow{ }^{t} c \cdot \chi^{*}(t, \lambda)^{-1} a=0 \quad(\text { for all } a \in A(t+\lambda)) \\
& \Leftrightarrow{ }^{t} c^{t} \cdot{ }^{t} \chi(t, \lambda) a=0 \quad(\text { for all } a \in A(t+\lambda)) \\
& \Leftrightarrow{ }^{t}(\chi(t, \lambda) c) \cdot a=0 \quad(\text { for all } a \in A(t+\lambda)) \\
& \Leftrightarrow \chi(t, \lambda) c \in A(t+\lambda)^{\perp} .
\end{aligned}
$$

Corollary. For any $\lambda$ in $\mathscr{L}$, we have

$$
A(t)^{\perp}=A(t+2 \lambda)^{\perp}
$$

as $g+1$-dimensional subspaces of $\mathbf{C}^{2^{g}}$.

Proof. We know from Proposition 7 that $c \in A(t)^{\perp}$ if and only if $\chi(t, 2 \lambda) c \in$ $A(t+2 \lambda)^{\perp}$. But since $\chi(t, 2 \lambda)$ is a scalar matrix, we have that $\chi(t, 2 \lambda) c \in A(t)^{\perp}$ if and only if $c \in A(t+2 \lambda)^{\perp}$. This completes the proof.

Now we define

$$
B=\bigcup A(t)^{\perp}, \quad t \in \mathbf{C}^{g} .
$$

By the above corollary, we can write this as

$$
B=\bigcup A(2 t)^{\perp}, \quad t \in J .
$$

Lemma 1. $B$ is an analytic subvariety of $\mathbf{C}^{2^{8}}$ of dimension at most $2 g+1$.

Proof. It was already noted that $\{A(t)\}$ form an analytic family of subspaces. This means that $\left\{A(t)^{\perp}\right\}$ is also a family of subspaces that vary analytically with $t$, as is easily seen through their definition (9). Now, for each $t \in \mathbf{C}^{g}$, we can view $A(2 t)^{\perp} \subset t \times \mathbf{C}^{2^{g}}$ so that we have

$$
\coprod_{t \in \mathbf{C}^{g}} A(2 t)^{\perp} \subset \mathbf{C}^{g} \times \mathbf{C}^{2^{g}} .
$$

By the above corollary, we have

$$
\coprod_{t \in J} A(2 t)^{\perp} \subset J \times \mathbf{C}^{2^{g}} .
$$

We can therefore define a new vector bundle $\mathscr{B}$ by

$$
E(\mathscr{B})=\coprod_{t \in J} A(2 t)^{\perp}
$$

Here $\mathscr{B}$ is a vector bundle of rank $g+1$ over $J$, and we have $E(\mathscr{B}) \subset E\left(\mathscr{J}_{2^{g}}\right)$, or $\mathscr{B} \hookrightarrow \mathscr{I}_{2^{8}}$. Also note that $E(\mathscr{B})$ has dimension $2 g+1$ as an analytic subvariety. It is clear that $B=p_{2}(E(\mathscr{B}))$ where $p_{2}: J \times \mathbf{C}^{2^{8}} \rightarrow \mathbf{C}^{2^{8}}$ is the 
projection onto the second factor. Now, for any compact set $K \subset \mathbf{C}^{2^{g}}$, we have that its inverse image is $p_{2}^{-1}(K)=J \times K$, which is clearly compact since $J$ is a compact space. Hence $p_{2}$ is a proper map. Then we may apply the proper mapping theorem which states that the image of an analytic subvariety under a proper map is again an analytic variety. Therefore $B$ is an analytic variety. Furthermore, the dimension of $B$ can be at most the dimension of $E(\mathscr{B})$, which is $2 g+1$. This completes the proof.

The following elementary proposition, whose proof we shall omit, will be used in the proof of the next lemma.

Proposition 8. Let $X$ and $Y$ be two linear subspaces of some complex vector space $\mathbf{C}^{N}$. Then

$$
X+Y=\mathbf{C}^{N} \quad \text { if and only if } X^{\perp} \cap Y^{\perp}=(0) .
$$

Lemma 2. Let $c_{1}, \ldots, c_{m} \in \mathbf{C}^{2^{8}}$. Then $\left\{\theta_{2}\left\langle c_{i} \mid 2 r-t\right\rangle(w(z))\right\}_{i=1, \ldots, m}$ will span $\Gamma\left(M, \rho_{t} \zeta^{2 g}\right)$ as functions of $z$ for all $t \in \mathbf{C}^{g}$ if and only if

$$
\left(\operatorname{Span}\left\{c_{1}, \ldots, c_{m}\right\}\right)^{\perp} \cap B=\{0\} .
$$

Proof. We first show the first implication. Suppose $c_{1}, \ldots, c_{m} \in \mathbf{C}^{2^{8}}$ are such that $\left\{\theta_{2}\left\langle c_{i} \mid 2 r-t\right\rangle(w(z))\right\}$ span $\Gamma\left(\rho_{t} \zeta^{2 g}\right)$ for all $t \in \mathbf{C}^{g}$. Fix any $t$. For any $c \in \mathbf{C}^{2^{8}}$, we have $\theta_{2}\langle c \mid 2 r-t\rangle(w(z)) \in \Gamma\left(\rho_{t} \zeta^{2 g}\right)$. Then

$$
\theta_{2}\langle c \mid 2 r-t\rangle(w(z))=\sum_{i=1}^{m} \alpha_{i} \theta_{2}\left\langle c_{i} \mid 2 r-t\right\rangle(w(z)),
$$

as functions of $z$, for some constants $\alpha_{i} \in \mathbf{C}$. This means that

$$
\theta_{2}\left\langle c-\sum \alpha_{i} c_{i} \mid 2 r-t\right\rangle(w(z))=0,
$$

for all $z$. Thus $c-\sum \alpha_{i} c_{i} \in A(t)$. This means that

$$
c \in A(t)+\operatorname{Span}\left\{c_{i}\right\} .
$$

Since $c \in \mathbf{C}^{2^{8}}$ was arbitrary, then

$$
A(t)+\operatorname{Span}\left\{c_{i}\right\}=\mathbf{C}^{2^{g}} .
$$

By Proposition 8 , then we have

$$
A(t)^{\perp} \cap \operatorname{Span}\left\{c_{i}\right\}^{\perp}=(0) .
$$

Since this is true for all fixed $t$, we have that $\bigcup A(t)^{\perp} \cap \operatorname{Span}\left\{c_{i}\right\}^{\perp}=(0)$, or

$$
B \cap \operatorname{Span}\left\{c_{i}\right\}^{\perp}=(0) .
$$

Now we prove the converse. Suppose $B \cap \operatorname{Span}\left\{c_{i}\right\}^{\perp}=(0)$. Then for any fixed $t \in \mathbf{C}^{g}$, we have $A(t)^{\perp} \cap \operatorname{Span}\left\{c_{i}\right\}^{\perp}=(0)$. By Proposition 8 , then we have $A(t)+\operatorname{Span}\left\{c_{i}\right\}=\mathbf{C}^{2^{g}}$. Now, take any element in $\Gamma\left(\rho_{t} \zeta^{2 g}\right)$; it is necessarily of 
the form $\theta_{2}\langle c \mid 2 r-t\rangle(w(z))$, for some $c \in \mathbf{C}^{2^{8}}$. Then $c \in A(t)+\operatorname{Span}\left\{c_{i}\right\}$ implies that $c=a+c^{\prime}$, for some $a \in A(t)$ and $c^{\prime} \in \operatorname{Span}\left\{c_{i}\right\}$. Then

$$
\theta_{2}\langle c \mid 2 r-t\rangle(w(z))=\theta_{2}\langle a \mid 2 r-t\rangle(w(z))+\theta_{2}\left\langle c^{\prime} \mid 2 r-t\right\rangle(w(z)) .
$$

But $a \in A(t)$ implies $\theta_{2}\langle a \mid 2 r-t\rangle(w(z)) \equiv 0$ in $z$. Hence

$$
\theta_{2}\langle c \mid 2 r-t\rangle(w(z))=\theta_{2}\left\langle c^{\prime} \mid 2 r-t\right\rangle(w(z)) .
$$

Thus any element in $\Gamma\left(\rho_{t} \zeta^{2 g}\right)$ can be written as $\theta_{2}\left\langle c^{\prime} \mid 2 r-t\right\rangle(w(z))$ for some $c^{\prime} \in \operatorname{Span}\left\{c_{i}\right\}$. This means that $\left\{\theta_{2}\left\langle c_{i} \mid 2 r-t\right\rangle(w(z))\right\}$ span $\Gamma\left(\rho_{t} \zeta^{2 g}\right)$. Since this is true for any $t \in \mathbf{C}^{g}$, the proof is complete.

We now state and prove our main result on the number of restricted second order theta functions it takes to span $\Gamma\left(\rho_{t} \zeta^{2 g}\right)$ for all $t$.

Theorem 2. For genus $g>2$ :

(1) There exist $2 g+1$ vectors $c_{1}, c_{2}, \ldots, c_{2 g+1} \in \mathbf{C}^{2^{g}}$ such that for all $t \in \mathbf{C}^{g}$, $\left\{\theta_{2}\left\langle c_{i} \mid 2 r-t\right\rangle(w(z))\right\}_{i=1, \ldots, 2 g+1}$ span $\Gamma\left(M, \rho_{t} \zeta^{2 g}\right)$ as functions of $z$;

(2) and $2 g+1$ is the minimum number necessary.

Proof. (1) By Lemma 2, we need to exhibit $c_{1}, \ldots, c_{2 g+1} \in \mathbf{C}^{2^{g}}$ such that

$$
\operatorname{Span}\left\{c_{1}, \ldots, c_{2 g+1}\right\} \cap B=(0) .
$$

Note that because $B$ is the union of linear spaces, $B$ has the property that for any $b \in B$, we also have $\alpha b \in B$ for any $\alpha \in \mathbf{C}$. That is, $B$ is a cone. Denote the standard map from $\mathbf{C}^{2^{8}} \backslash(0)$ to $\mathbf{C P}^{2^{8}-1}$ by

$$
\pi: \mathbf{C}^{2^{8}} \backslash(0) \rightarrow \mathbf{C P}^{2^{8}-1} .
$$

Then denote $\mathbf{P} B=\pi(B \backslash(0))$. By Lemma $1, B$ is an analytic subvariety of $\mathbf{C}^{2^{8}}$. Then $\mathbf{P B}$ is also an analytic subvariety of $\mathbf{C P}^{2^{8}-1}$. The fact that $B$ is a cone means that $\pi^{-1}(\mathbf{P} B)=B \backslash(0)$. This implies that

$$
\operatorname{dim} \mathbf{P} B=\operatorname{dim} B-1 .
$$

Now, a generic linear subvariety of $\mathbf{C} \mathbf{P}^{2^{8}-1}$ of dimension less than $2^{g}-1-$ $\operatorname{dim} \mathbf{P} B$ will not intersect $\mathbf{P} B$. That is, for a generic linear subspace $X \subset \mathbf{C}^{2^{8}}$ of dimension $2^{g}-1-\operatorname{dim} \mathbf{P} B, \mathbf{P} X=\pi(X \backslash(0)) \subset \mathbf{C P}^{2^{g}-1}$ will have dimension $2^{g}-1-\operatorname{dim} \mathbf{P} B-1$ and so

$$
\mathbf{P} X \cap \mathbf{P} B=\varnothing .
$$

This means that $X \cap B=(0)$. Pick in particular one such subspace $X$ of dimension $2^{g}-1-\operatorname{dim} \mathbf{P} B$. Let $W=X^{\perp}$. Then $W^{\perp}=X$ so that $W^{\perp} \cap B=$ (0). But $\operatorname{dim} W=2^{g}-\operatorname{dim} X=1+\operatorname{dim} \mathbf{P} B=\operatorname{dim} B$. Hence we can express $W=\operatorname{Span}\left\{c_{1}, \ldots, c_{\text {cim } B}\right\}$, for some choices $c_{i} \in \mathbf{C}^{2^{g}}$. Since $\operatorname{dim} B \leq 2 g+1$ by Lemma 1 , we can write $W=\operatorname{Span}\left\{c_{1}, \ldots, c_{2 g+1}\right\}$. Then

$$
\operatorname{Span}\left\{c_{1}, \ldots, c_{2 g+1}\right\}^{\perp} \cap B=(0) \text {. }
$$

This completes the proof of part (1). 
(2) If it were possible for $c_{1}, \ldots, c_{m}$ to satisfy the theorem for $m \leq 2 g$, then trivially it could be done with $m=2 g$. Hence to show that $2 g+1$ is the minimum necessary, we only need to show that it is not possible for any $\left\{c_{1}, \ldots, c_{2 g}\right\}$ to satisfy the theorem. Suppose by contradiction that there do exist $\left\{c_{1}, \ldots, c_{2 g}\right\}$ that satisfy the theorem. As in Proposition 4, any $\theta_{2}\langle c \mid 2 r-2 t\rangle(w)$ picks out a line subbundle $\mu_{c}^{*}$ of $\widetilde{\chi^{*}}$. Following the idea in Remark 1 , consider the vector bundle of rank $2 g$,

$$
\mu^{*}=\bigoplus_{i=1}^{2 g} \mu_{c_{i}}^{*}
$$

so

$$
E\left(\mu^{*}\right)=\coprod\left(\bigoplus_{i=1}^{2 g} \operatorname{Span}\left\{\theta_{2}\left\langle c_{i} \mid 2 r-2 t\right\rangle(w)\right\}\right) /(\sim) .
$$

Look at the map

$$
\mu^{*} \rightarrow \widetilde{\chi^{g+1^{*}}}
$$

which on each fiber is the linear map

$$
\bigoplus_{i=1}^{2 g} \operatorname{Span}\left\{\theta_{2}\left\langle c_{i} \mid 2 r-2 t\right\rangle(w)\right\} \rightarrow \Gamma\left(\rho_{2 t} \zeta^{2 g}\right)
$$

given by the restriction

$$
\theta_{2}\left\langle c_{i} \mid 2 r-2 t\right\rangle(w) \mapsto \theta_{2}\left\langle c_{i} \mid 2 r-2 t\right\rangle(w(z)) .
$$

The assumption that $\left\{\theta_{2}\left\langle c_{i} \mid 2 r-2 t\right\rangle(w(z))\right\}$ spans $\Gamma\left(\rho_{2 t} \zeta^{2 g}\right)$ for every $t \in \mathbf{C}^{g}$ means that this map of bundles is surjective on each fiber. Hence we have a surjective map of vector bundles $\mu^{*} \rightarrow \widetilde{\chi^{g+1^{*}}}$. This is equivalent to having an injective map of the dual bundles $\chi^{g+1} \rightarrow \mu$. Let $Q$ be the quotient bundle. So we have an exact sequence

$$
0 \rightarrow \widetilde{\chi^{g+1}} \rightarrow \mu \rightarrow Q \rightarrow 0 .
$$

Now, $Q$ is a bundle of rank $2 g-(g+1)=g-1$. Hence its $g$ th Chern class $c_{g}(Q)$ must be zero. Now we compute the Chern class of $Q$. From (12) we have that

$$
c(Q)=c(\mu) / c\left(\widetilde{\chi^{g+1}}\right) .
$$

We know from previous discussions that $c\left(\widetilde{\chi^{g+1}}\right)=e^{4 \theta}$. From (11) we see that $c\left(\mu^{*}\right)=\prod_{i=1}^{2 g} c\left(\mu_{c_{i}}^{*}\right)$, which by Proposition 5 implies $c\left(\mu^{*}\right)=(1-2 \theta)^{2 g}$. Then we have that $c(\mu)=(1+2 \theta)^{2 g}$. Consequently, we arrive at

$$
c(Q)=(1+2 \theta)^{2 g} / e^{4 \theta}=(1+2 \theta)^{2 g} e^{-4 \theta} .
$$


Then the $g$ th Chern class of $Q$ is

$$
\begin{aligned}
c_{g}(Q) & =\sum_{j+k=g}\left(\begin{array}{c}
2 g \\
j
\end{array}\right)(2 \theta)^{j} \cdot \frac{(-4 \theta)^{k}}{k !} \\
& =\sum_{j+k=g}\left(\begin{array}{c}
2 g \\
j
\end{array}\right) \frac{(-2)^{k}(2 \theta)^{k}(2 \theta)^{j}}{k !} \\
& =(2 \theta)^{g} \sum_{j+k=g}\left(\begin{array}{c}
2 g \\
j
\end{array}\right) \frac{(-2)^{k}}{k !} \\
& =(2 \theta)^{g} \sum_{k=0}^{g}\left(\begin{array}{c}
2 g \\
g-k
\end{array}\right) \frac{(-2)^{k}}{k !} \\
& =(2 \theta)^{g} \sum_{k=0}^{g} \frac{(2 g) !}{(g-k) !(g+k) !} \frac{(-2)^{k}}{k !} \\
& =\frac{(2 \theta)^{g}}{g !} \sum_{k=0}^{g} \frac{(2 g) !}{(g+k) !} \frac{g !}{(g-k) ! k !}(-2)^{k} \\
& =\frac{(2 \theta)^{g}}{g !} \sum_{k=0}^{g} \frac{(2 g) !}{(g+k) !}\left(\begin{array}{l}
g \\
k
\end{array}\right)(-2)^{k} .
\end{aligned}
$$

Since $c_{g}(Q)=0$ and $\frac{(2 \theta)^{g}}{g !} \neq 0 \in H^{2 g}(J, \mathbf{Z}) \cong \mathbf{Z}$, we must have

$$
\sum_{k=0}^{g} \frac{(2 g) !}{(g+k) !}\left(\begin{array}{l}
g \\
k
\end{array}\right)(-2)^{k}=0 \text {. }
$$

Separating out the terms for $k=g$ and $k=g-1$, we have

$$
(-2)^{g}+(2 g) g(-2)^{g-1}+\sum_{k=0}^{g-2} \frac{(2 g) !}{(g+k) !}\left(\begin{array}{l}
g \\
k
\end{array}\right)(-2)^{k}=0 .
$$

Note that for $k=0,1, \ldots, g-2$, we have that $g+k \leq 2 g-2$ and thus

$$
(2 g-1) \text { divides } \frac{(2 g) !}{(g+k) !} \text {. }
$$

This implies that

$$
(2 g-1) \text { divides } \sum_{k=0}^{g-2} \frac{(2 g) !}{(g+k) !}\left(\begin{array}{l}
g \\
k
\end{array}\right)(-2)^{k},
$$

and so we must have that

$$
(2 g-1) \text { divides }(-2)^{g}+(2 g) g(-2)^{g-1},
$$

which is the same as

$$
(2 g-1) \text { divides }(-2)^{g-1}\left(-2+2 g^{2}\right) .
$$


Since $(2 g-1)$ is an odd number, we must have that

$$
(2 g-1) \text { divides }\left(2 g^{2}-2\right) \text {. }
$$

Note $2 g^{2}-2=g(2 g-1)+g-2$. Consequently, it must be true that

$$
(2 g-1) \text { divides }(g-2) \text {. }
$$

But since we are assuming that $g>2$, we have that both $(2 g-1)$ and $(g-2)$ are positive numbers with $(2 g-1)>(g-2)$. So it is impossible to have that $(2 g-1)$ divides $(g-2)$, and we have arrived at a contradiction. This completes the proof of the theorem.

\section{ONE APPLICATION}

As one application, consider the subvariety of special positive divisors in $J$,

$$
W_{r}^{n}=\left\{t \in \mathbf{C}^{g}: \operatorname{dim} \Gamma\left(\rho_{t} \zeta^{r}\right)>n\right\} / \mathscr{L} .
$$

Gunning has shown in [6] and [7] that they can be described by

$$
W_{m-2}^{m-p}-\alpha=\left\{t \in \mathbf{C}^{g}: \operatorname{rank}\left\{\theta_{2}[\nu \mid 2 r-t]\left(w\left(a_{j}\right)\right)\right\}_{\nu \in \mathbf{Z}^{8} / 2 \mathbf{Z}^{8} ; j=1, \ldots, m}<p\right\} / \mathscr{L}
$$

for any distinct points $a_{1}, \ldots, a_{m} \in M$ and where $\alpha=w\left(a_{1}\right)+\cdots+w\left(a_{m}\right)$. Theorem 2 says that we can replace this with

Theorem 3. There exist $c_{1}, \ldots, c_{2 g+1} \in \mathbf{C}^{2^{8}}$ such that

$$
W_{m-2}^{m-p}-\alpha=\left\{t \in \mathbf{C}^{g}: \operatorname{rank}\left\{\theta_{2}\left\langle c_{i} \mid 2 r-t\right\rangle\left(w\left(a_{j}\right)\right)\right\}_{i=1, \ldots, 2 g+1 ; j=1, \ldots, m}<p\right\} / \mathscr{L}
$$

for any distinct points $a_{1}, \ldots, a_{m} \in M$ and where $\alpha=w\left(a_{1}\right)+\cdots+w\left(a_{m}\right)$.

Proof. By Theorem 2, take $c_{1}, \ldots, c_{2 g+1} \in \mathbf{C}^{2^{8}}$ such that

$$
\left\{\theta_{2}\left\langle c_{i} \mid 2 r-t\right\rangle(w(z))\right\}_{i=1}^{2 g+1}
$$

spans $\Gamma\left(\rho_{t} \zeta^{2 g}\right)$ for each $t \in \mathbf{C}^{g}$. This means that for each $t$, the span of $\left\{\theta_{2}\left\langle c_{i} \mid 2 r-t\right\rangle(w(z))\right\}_{i=1}^{2 g+1}$ is the same as the span of

$$
\left\{\theta_{2}[\nu \mid 2 r-t](w(z))\right\}_{\nu \in \mathbf{Z}^{8} / 2 \mathbf{Z}^{8}},
$$

viewed as functions of $z$. Considering these two sets as column vectors, this means that for each fixed $t$, there is a $2^{g} \times 2 g+1$ matrix $A$ and a $2 g+1 \times 2^{g}$ matrix $B$ such that

$$
\begin{aligned}
& \left\{\theta_{2}[\nu \mid 2 r-t](w(z))\right\}_{\nu}=A\left\{\theta_{2}\left\langle c_{i} \mid 2 r-t\right\rangle(w(z))\right\}_{i}, \\
& \left\{\theta_{2}\left\langle c_{i} \mid 2 r-t\right\rangle(w(z))\right\}_{i}=B\left\{\theta_{2}[\nu \mid 2 r-t](w(z))\right\}_{\nu} .
\end{aligned}
$$

In particular these equations are true for each $z=a_{i}$, so we have

$$
\begin{aligned}
& \left\{\theta_{2}[\nu \mid 2 r-t]\left(w\left(a_{j}\right)\right)\right\}_{\nu, j}=A\left\{\theta_{2}\left\langle c_{i} \mid 2 r-t\right\rangle\left(w\left(a_{j}\right)\right)\right\}_{i, j}, \\
& \left\{\theta_{2}\left\langle c_{i} \mid 2 r-t\right\rangle\left(w\left(a_{j}\right)\right)\right\}_{i, j}=B\left\{\theta_{2}[\nu \mid 2 r-t]\left(w\left(a_{j}\right)\right)\right\}_{\nu, j} .
\end{aligned}
$$


These imply that

$$
\begin{aligned}
& \operatorname{rank}\left\{\theta_{2}[\nu \mid 2 r-t]\left(w\left(a_{j}\right)\right)\right\}_{\nu, j} \leq \operatorname{rank}\left\{\theta_{2}\left\langle c_{i} \mid 2 r-t\right\rangle\left(w\left(a_{j}\right)\right)\right\}_{i, j}, \\
& \operatorname{rank}\left\{\theta_{2}\left\langle c_{i} \mid 2 r-t\right\rangle\left(w\left(a_{j}\right)\right)\right\}_{i, j} \leq \operatorname{rank}\left\{\theta_{2}[\nu \mid 2 r-t]\left(w\left(a_{j}\right)\right)\right\}_{\nu, j} .
\end{aligned}
$$

Therefore,

$$
\operatorname{rank}\left\{\theta_{2}\left\langle c_{i} \mid 2 r-t\right\rangle\left(w\left(a_{j}\right)\right)\right\}_{i, j}=\operatorname{rank}\left\{\theta_{2}[\nu \mid 2 r-t]\left(w\left(a_{j}\right)\right)\right\}_{\nu, j},
$$

and so

$$
\begin{aligned}
\left\{t \in \mathbf{C}^{g}: \operatorname{rank}\left\{\theta_{2}\left\langle c_{i} \mid 2 r-t\right\rangle\left(w\left(a_{j}\right)\right)\right\}_{i, j}<p\right\} \\
\quad=\left\{t \in \mathbf{C}^{g}: \operatorname{rank}\left\{\theta_{2}[\nu \mid 2 r-t]\left(w\left(a_{j}\right)\right)\right\}_{\nu, j}<p\right\} .
\end{aligned}
$$

Then by equation (14), we are done.

We have replaced the $2^{g} \times m$ matrix with a smaller $2 g+1 \times m$ matrix. In particular, for $m=p=3$, we have $W_{m-2}^{m-p}=W_{1}^{0}=M$, the image of the curve in the Jacobian, and so

$$
\begin{aligned}
M-\alpha & =W_{1}^{0}-\alpha \\
& =\left\{t \in \mathbf{C}^{g}: \operatorname{rank}\left\{\theta_{2}\left\langle c_{i} \mid 2 r-t\right\rangle\left(w\left(a_{j}\right)\right)\right\}_{i=1, \ldots, 2 g+1 ; j=1,2,3}<3\right\} / \mathscr{L} .
\end{aligned}
$$

Therefore $M-\alpha$, a translate of the curve, can be recovered as the common zeroes of the $\left(\begin{array}{c}2 g+1 \\ 3\end{array}\right)=\frac{1}{3}\left(4 g^{3}-g\right)$ sets of $3 \times 3$ subdeterminants of the $2 g+1 \times 3$ matrix

$$
\left\{\theta_{2}\left\langle c_{i} \mid 2 r-t\right\rangle\left(w\left(a_{j}\right)\right)\right\}_{i=1, \ldots, 2 g+1 ; j=1,2,3} .
$$

It is amusing to note that the description of subvarieties of special positive divisors in Theorem 3 is reminiscent of the description of tangent cones to the same subvarieties in Kempf's singularity theorem [13]. Both descriptions use certain minors of a certain matrix to generate the necessary equations.

\section{SOME COMMENTS}

One aim of this paper was to find in some sense a small explicitly describable bundle in which the Picard bundle sits. We can say something about $\widetilde{\chi^{g+1}}$, the pullback of the Picard bundle by the doubling map. By Theorem 2, we have shown that $\widetilde{\chi^{g+1}}$ sits inside a rank $2 g+1$ scalar vector bundle,

$$
\widetilde{\chi^{g+1}} \hookrightarrow \rho_{2 r} \xi^{2} \mathscr{F}_{2 g+1} \text {. }
$$

An interpretation of Theorem 2 is that there exists an imbedding

$$
\rho_{-2 r} \xi^{-2} \widetilde{\chi^{g+1}} \hookrightarrow \mathscr{I}_{2 g+1},
$$

and that $\mathscr{J}_{2 g+1}$ is the smallest trivial bundle we can use. This imbedding is not unique and depends on which suitable $c_{1}, \ldots, c_{2 g+1}$ one chooses. 
It would be nice to know exactly which $c_{1}, \ldots, c_{2 g+1}$ we can use in Theorem 2. Handling such a question would involve the study of $B$ as defined in equation (10) because by Lemma 2 , such vectors are characterized by

$$
\operatorname{Span}\left\{c_{1}, \ldots, c_{2 g+1}\right\}^{\perp} \cap B=(0) .
$$

Of course this $B \subset \mathbf{C}^{2^{8}}$ depends on $M$.

Lemma 3. For each $M$, the corresponding $B$ is an analytic subvariety of $\mathbf{C}^{2^{8}}$ of dimension exactly $2 g+1$.

Proof. From the proof of Theorem 2, part (1), there exists a linear subspace $W \subset \mathbf{C}^{2^{8}}$ with $\operatorname{dim} W=\operatorname{dim} B$ such that $W^{\perp} \cap B=(0)$. Taking $W=$ $\operatorname{Span}\left\{c_{1}, \ldots, c_{\operatorname{dim} B}\right\}$, we have $\operatorname{Span}\left\{c_{1}, \ldots, c_{\operatorname{dim} B}\right\} \cap B=(0)$, which by Lemma 2 implies that $c_{1}, \ldots, c_{\operatorname{dim} B}$ satisfy Theorem 2, part (1). Since Theorem 2, part (2) says $2 g+1$ is the minimal number, we must have $\operatorname{dim} B \geq$ $2 g+1$. Since Lemma 1 says that $B$ is an analytic variety with $\operatorname{dim} B \leq 2 g+1$, we conclude that $\operatorname{dim} B=2 g+1$.

Corollary. $\mathbf{P} B$ is an analytic subvariety of $\mathbf{C} \mathbf{P}^{2^{8}-1}$ of dimension $2 g$.

A natural question is what is the cohomology class of $\mathbf{P} B$, that is, what is its degree $b=[\mathbf{P} B] \in H^{2 i}\left(\mathbf{P}^{2^{8}-1}, \mathbf{Z}\right) \cong \mathbf{Z}$, where $i=2^{g}-2 g-1$. Thus to each $M$ is associated this positive integer $b(M)$. It would be interesting to see if this number varies with $M$. We can attempt to calculate this number $b=[\mathbf{P} B]$ as follows. Take a generic linear subspace $H \subset \mathbf{C}^{2^{8}}$ of dimension $2^{g}-2 g$, so that $X=\mathbf{P} H \cap \mathbf{P} B$ is a finite set of points, namely $b$ number of points. Now recall the bundle $\mathscr{B}$ that was defined in the proof of lemma 1. We have $E(\mathscr{B}) \subset J \times \mathbf{C}^{2^{8}}$, so $E(\mathbf{P} \mathscr{B}) \subset J \times \mathbf{P C}^{2 g-1}$ where $\mathbf{P} \mathscr{B}$ is the associated projective bundle. We have $p_{2}: E(\mathbf{P} \mathscr{B}) \rightarrow \mathbf{P} B$, a surjective map between two compact varieties of the same dimension, $2 g$, so it is a finite map. Let $d=$ degree of $p_{2}$. For a generic $H, X$ will be a generic finite set in $\mathbf{P} B$ and so $p_{2}^{-1}(X)$ will be a finite set consisting of $b d$ number of points. Consider $X^{\prime}=p_{1}\left(p_{2}^{-1}(X)\right) \subset J$. Note that $p_{1}$ restricted to $p_{2}^{-1}(X) \rightarrow X^{\prime}$ must be a one-to-one map. For suppose $p_{2}\left(t, c_{1}\right), p_{2}\left(t, c_{2}\right) \in X=\mathbf{P} B \cap \mathbf{P} H$. This means that $c_{1}, c_{2} \in \mathbf{P} A(t)^{\perp}$, which implies that $\operatorname{Span}\left\{c_{1}, c_{2}\right\} \subset \mathbf{P} B$. Since $\operatorname{Span}\left\{c_{1}, c_{2}\right\} \subset \mathbf{P} H$, then also $\operatorname{Span}\left\{c_{1}, c_{2}\right\} \subset X$. Since $X$ is a finite set this forces $c_{1}=c_{2}$. Hence $X^{\prime}$ consists of $b d$ number of points. Noting that [one point] $=\frac{\theta^{g}}{g !}$, this means

$$
\left[X^{\prime}\right]=b d \frac{\theta^{g}}{g !} \in H^{2 g}(J, \mathbf{Z}),
$$

Now, $X^{\prime}$ consists exactly of those points $t$ for which $H \cap A(t)^{\perp} \neq(0)$. By the proof to Lemma 2 , this is precisely where $\left\{\theta_{2}\langle c \mid 2 r-t\rangle(w(z)): c \in H^{\perp}\right\}$ fails to span $\Gamma\left(M, \rho_{t} \zeta^{2 g}\right)$. Pick some basis $c_{1}, \ldots, c_{2 g}$ of $H^{\perp}$. Then $X^{\prime}$ is 
where the map $\mu^{*}=\mu_{c_{1}}^{*} \oplus \cdots \oplus \mu_{c_{2 g}}^{*} \rightarrow{\widetilde{\chi^{g+1}}}^{*}$ is not surjective. Looking at the dual of this map, $X^{\prime}$ is where $\widetilde{\chi^{g+1}} \rightarrow \mu$ fails to be injective. The expected dimension of the degeneracy locus is $\operatorname{dim} \widetilde{J}-(g+1-g)(2 g-g)=0$, and $X^{\prime}$ has precisely this dimension. So we can calculate the class of $X^{\prime}$ by Porteous' formula:

$$
\begin{aligned}
{\left[X^{\prime}\right] } & =\Delta_{2 g-g, g+1-g}\left(c(\mu) / c\left(\widetilde{\chi^{g+1}}\right)\right) \\
& =\Delta_{g, 1}\left((1+2 \theta)^{2 g} / e^{4 \theta}\right) \\
& =g \text { th term of }(1+2 \theta)^{2 g} e^{-4 \theta} \\
& =\sum_{j+k=g}\left(\begin{array}{c}
2 g \\
j
\end{array}\right)(2 \theta)^{j} \cdot \frac{\left.(-4 \theta)^{k}\right)}{k !} .
\end{aligned}
$$

From the calculation in (13), this can be simplified to

$$
\left[X^{\prime}\right]=\frac{2^{g} \theta^{g}}{g !} \sum_{k=0}^{g} \frac{(2 g) !}{(g+k) !}\left(\begin{array}{l}
g \\
k
\end{array}\right)(-2)^{k} .
$$

Comparing this equation to equation (15), we obtain that

$$
b d=2^{g} \sum_{k=0}^{g} \frac{(2 g) !}{(g+k) !}\left(\begin{array}{l}
g \\
k
\end{array}\right)(-2)^{k} .
$$

In particular, we have

Proposition 9. The number $b(M)=[\mathbf{P} B]$ divides the number

$$
2^{g} \sum_{k=0}^{g} \frac{(2 g) !}{(g+k) !}\left(\begin{array}{l}
g \\
k
\end{array}\right)(-2)^{k} .
$$

In particular, there is only a finite number of possible values for $b(M)$, namely the factors of the above number.

It might still turn out to be the case that $b(M)$ is the same for every compact marked Riemann surface $M$.

The vector bundle $\mathscr{B}$ seems to be involved in this question. We can say one thing about this bundle.

Proposition 10. $\mathscr{B} \cong \rho_{-2 r} \xi^{-2} \otimes \widetilde{\chi^{g+1}}$.

Proof. By definition, $E(\mathscr{B})=\bigsqcup_{J} A(2 t)^{\perp}$. Since

$$
A(2 t)=\left\{c \in \mathbf{C}^{2^{8}}:{ }^{t} \mathcal{c} \cdot \vec{\theta}_{2}[2 r-2 t](w(z)) \equiv 0 \text { in } z\right\},
$$

it is clear that $A(2 t)^{\perp}=\operatorname{Span}\left\{\vec{\theta}_{2}[2 r-2 t](w(z)): z \in \widetilde{M}\right\}$. Let $N: \chi^{g+1} \hookrightarrow \chi$ be given by the $2^{g} \times g+1$ matrices $N(t)$. This means $\vec{\theta}_{2}[2 r-2 t](w(z))=$ 
$N(2 t) \vec{\theta}^{g+1}(2 t, z)$, where $\vec{\theta}^{g+1}(2 t, z)$ is the vector of generalized theta functions. So $A(2 t)^{\perp}=\operatorname{Span}\left\{N(2 t) \vec{\theta}^{g+1}(2 t, z): z \in \widetilde{M}\right\}$. Since $\operatorname{Span}\left\{\vec{\theta}^{g+1}(2 t, z)\right.$ : $z \in \widetilde{M}\}=\mathbf{C}^{g+1}$, we have $A(2 t)^{\perp}=\operatorname{Span}\left\{N(2 t) a: a \in \mathbf{C}^{g+1}\right\} \subset \mathbf{C}^{2^{g}}$. Thus

$$
E(\mathscr{B})=\coprod_{t \in J} N(2 t) \mathbf{C}^{g+1} \subset J \times \mathbf{C}^{2^{8}} .
$$

Then $\rho_{2 r} \xi^{2} \otimes \mathscr{B}$ would have total space

$$
\begin{aligned}
E\left(\rho_{2 r} \xi^{2} \otimes \mathscr{B}\right) & =\coprod_{t \in \mathbf{C}^{g}} N(2 t) \mathbf{C}^{g+1} /(\sim) \\
& \subset E\left(\rho_{2 r} \xi^{2} \otimes \mathscr{I}_{2^{g}}\right)=E(\tilde{\chi}) .
\end{aligned}
$$

The image of $\widetilde{\chi^{g+1}} \hookrightarrow \tilde{\chi}$ on each fiber over $t \in \mathbf{C}^{g}$ is $N(2 t) \mathbf{C}^{g+1} \subset \mathbf{C}^{2^{g}}$, which is exactly the fiber of $\rho_{2 r} \xi^{2} \otimes \mathscr{B}$ over $t$. So we really get a map $\widetilde{\chi^{g+1}} \hookrightarrow$ $\rho_{2 r} \xi^{2} \otimes \mathscr{B} \subset \tilde{\chi}$. This is necessarily an isomorphism since it is an injective map between two bundles of the same rank, so we have $\rho_{2 r} \xi^{2} \otimes \mathscr{B} \cong \widetilde{\chi^{g+1}}$, which completes the proof.

Using $c\left(\rho_{-2 r} \xi^{-2}\right)=1-2 \theta$ and $c\left(\widetilde{\chi^{g+1}}\right)=e^{4 \theta}$ and the formula for the Chern class of the tensor product of a vector bundle with a line bundle, we get

Corollary. The nth Chern class of $\mathscr{B}$ is

$$
c_{n}(\mathscr{B})=\sum_{k=0}^{n}\left(\begin{array}{c}
g+1-k \\
n-k
\end{array}\right) \frac{(-2)^{g+k}}{k !} \theta^{n} .
$$

This completes our discussion on the subvariety $B \subset \mathbf{C}^{2^{8}}$.

Next we wish to consider the set of subspaces $\operatorname{Span}\left\{c_{1}, \ldots, c_{2 g+1}\right\}$ that satisfy the main theorem. Denote by $\mathscr{Y}$ the set of $2 g+1$-dimensional subspaces $Y=\operatorname{Span}\left\{c_{1}, \ldots, c_{2 g+1}\right\}$ of $\mathbf{C}^{2^{8}}$ that satisfy Theorem 2 . So $\mathscr{Y}$ is a subset of the Grassmannian $\operatorname{Gr}\left(2 g+1,2^{g}\right)$.

Proposition 11. The set $\mathscr{Y}$ is a Zariski open subset of $\operatorname{Gr}\left(2 g+1,2^{g}\right)$.

Proof. Denote for simplicity $\mathrm{Gr}=\mathrm{Gr}\left(2 g+1,2^{g}\right)$. Of course $\mathscr{Y}$ is nonempty by Theorem 2. Let $\mathscr{Z}=\mathrm{Gr}-\mathscr{Y}$ be the complement of $\mathscr{Y}$. We have to show that $\mathscr{Z}$ is an analytic subvariety of $\mathrm{Gr}$. By Lemma $2, Y \in \mathscr{Y}$ if and only if $Y^{\perp} \cap B=(0)$, so we have

$$
\begin{aligned}
\mathscr{Z} & =\left\{Y: Y^{\perp} \cap B \neq(0)\right\} \\
& =\left\{Y: Y^{\perp} \cap \bigcup_{t \in J} A(2 t)^{\perp} \neq(0)\right\} \\
& =\bigcup_{t \in J}\left\{Y: Y^{\perp} \cap A(2 t)^{\perp} \neq(0)\right\} \\
& =\bigcup_{t \in J}\left\{Y: Y+A(2 t) \neq \mathbf{C}^{2^{g}}\right\} .
\end{aligned}
$$


Consider the set

$$
\mathscr{Z}^{\prime}=\coprod_{t \in J}\left\{Y: Y+A(2 t) \neq \mathbf{C}^{2^{8}}\right\} \subset J \times \mathrm{Gr} .
$$

At each point $\left(t_{0}, Y_{0}\right) \in J \dot{\times} \mathrm{Gr}$, there is a neighborhood which can be parametrized by $\left(t, \operatorname{Span}\left\{y_{1}, \ldots, y_{2 g+1}\right\}\right)$, where $y_{1}, \ldots, y_{2 g+1}$ are vectors in $\mathbf{C}^{2^{8}}$ sufficiently near some fixed basis $y_{1}^{0}, \ldots, y_{2 g+1}^{0}$ of $Y_{0}$. And restricting $t$ close to $t_{0}$, there exists some parametrization $A(2 t)=\operatorname{Span}\left\{a_{1}(t), \ldots, a_{n}(t)\right\}$, where $n=2^{g}-(g+1)$. Then $\mathscr{Z}^{\prime}$ restricted to this neighborhood of $\left(t_{0}, Y_{0}\right)$ is exactly where

$$
\operatorname{rank}\left\{y_{1}, \ldots, y_{2 g+1}, a_{1}(t), \ldots, a_{n}(t)\right\}<2^{g},
$$

and so $\mathscr{Z}^{\prime}$ is the set of common zeroes of all the $2^{g} \times 2^{g}$ subdeterminants of this matrix. This means that locally in $J \times \mathrm{Gr}, \mathscr{Z}^{\prime}$ is an analytic subvariety, and hence $\mathscr{Z}^{\prime}$ is an analytic subvariety. Note $\mathscr{Z}=p_{2}\left(\mathscr{Z}^{\prime}\right)$, where $p_{2}: J \times \mathrm{Gr} \rightarrow \mathrm{Gr}$ is the projection onto the second factor. Since $J$ is compact, $p_{2}$ is a proper map. Therefore $\mathscr{Z}$, being the image of a subvariety under a proper map, is an analytic subvariety of $\mathrm{Gr}$. This completes the proof.

Lemma 4. The set $\mathscr{Y}$ is the complement of a codimension 1 subvariety in the Grassmannian $\operatorname{Gr}\left(2 g+1,2^{g}\right)$.

Proof. We will be using the fact that the dimension of a Grassmannian $\operatorname{Gr}(k, n)$ is $(n-k) k$. Let $\mathscr{Z}$ be the complement of $\mathscr{Y}$ as above. We know that $\mathscr{Z}$ is a subvariety, and that

$$
\mathscr{Z}=\left\{Y: Y^{\perp} \cap B \neq(0)\right\} .
$$

We will count the dimension of $\mathscr{Z}$ by counting the number of ways of choosing $Y^{\perp} \in \operatorname{Gr}\left(2^{g}-2 g-1,2^{g}\right)$. The only requirement is that $Y^{\perp}$ must intersect $B$. Since $B$ is a cone of dimension $2 g+1$, the number of different ways of $Y^{\perp}$ intersecting $B$ in a line is of dimension $2 g$. Having chosen a line $L$ to be contained in $Y^{\perp}$, we need to fill the rest of $Y^{\perp}$ by choosing

$$
Y^{\perp} / L \in \operatorname{Gr}\left(2^{g}-2 g-2, \mathrm{C}^{2^{g}} / L\right) \text {. }
$$

The dimension of $\operatorname{Gr}\left(2^{g}-2 g-2,2^{g}-1\right)$ is $\left(2^{g}-2 g-2\right)(2 g+1)$. Thus the dimension of $\mathscr{Z}$ is

$$
2 g+\left(2^{g}-2 g-2\right)(2 g+1)=\left(2^{g}-2 g-1\right)(2 g+1)-1 .
$$

Since the dimension of $\operatorname{Gr}\left(2 g+1,2^{g}\right)$ is $\left(2^{g}-2 g-1\right)(2 g+1)$, we have that $\mathscr{Z}$ is of codimension 1 . This completes the proof.

\section{Generalizations}

We offer two generalization of the main theorem, one to $d$ th order theta functions, the other to arbitrary vector bundles. 
Theorem 4. For genus $g>1$ and $d>2$ :

(1) There exists $d g+1$ vectors $c_{1}, c_{2}, \ldots, c_{d g+1} \in \mathbf{C}^{d^{g}}$ such that for all $t \in \mathbf{C}^{g},\left\{\theta_{d}\left\langle c_{i} \mid 2 r-t\right\rangle(w(z))\right\}_{i=1, \ldots, d g+1}$ span $\Gamma\left(M, \rho_{t} \zeta^{d g}\right)$ as functions of $z$;

(2) and $d g+1$ is the minimum number necessary.

Theorem 5. Let $\mathscr{A}$ be a (holomorphic) vector bundle of rank $n$ over a compact analytic variety of dimension $k$. Suppose there is an imbedding $\mathscr{A} \hookrightarrow \mathscr{J}_{N}$, for some $N \geq n+k$, where $\mathscr{I}_{N}$ denotes the trivial bundle of rank $N$. Then:

(1) There exists an imbedding $\mathscr{A} \hookrightarrow \mathscr{I}_{n+k}$.

(2) If in addition the kth Segre class of $\mathscr{A}, s_{k}(\mathscr{A})$, is nonzero, then $n+k$ is the minimum number necessary.

Proof of Theorem 4.

(1) The proof is analogous to the proof of Theorem 2 except for some slight differences. We define a rank $d^{g}$ factor of automorphy $\chi_{d}$ as that for which

$$
\theta_{d}\langle c \mid d r-(t+\lambda)\rangle(w)=\rho_{w}(\lambda) \chi_{d}(t, \lambda) \theta_{d}\langle c \mid d r-t\rangle(w) .
$$

Precisely, we have that for $\lambda=p+\Omega q \in \mathscr{L}$,

$$
\chi_{d}(t, \lambda)=\rho_{r}(\lambda) \exp \frac{-2 \pi i}{d}^{t} q\left(t+\frac{1}{2} \Omega q\right) \chi_{d}(\lambda),
$$

where $\chi_{d}(\lambda)$ is a $d^{g} \times d^{g}$ matrix with entries

$$
\chi_{d}(\lambda)_{\nu \mu}=\delta_{\nu-q}^{\mu} \cdot \exp \frac{-2 \pi i}{d}{ }^{t} p \cdot \nu .
$$

Its dual $\chi_{d}^{*}$ has total space

$$
E\left(\chi_{d}^{*}\right)=\coprod_{t \in J} \Gamma\left(\rho_{t-d r} \xi^{d}\right)
$$

with the natural identification of $\Gamma\left(\rho_{t-d r} \xi^{d}\right)$ with $\Gamma\left(\rho_{t+\lambda-d r} \xi^{d}\right)$. Let

$$
A(t)=\left\{c \in \mathbf{C}^{d^{g}}: \theta_{d}\langle c \mid d r-t\rangle(w(z)) \equiv 0 \text { in } z\right\} \subset \mathbf{C}^{d^{g}},
$$

which is in a sense the kernel of the surjection $\Gamma\left(\rho_{t-d r} \xi^{d}\right) \rightarrow \Gamma\left(\rho_{t} \zeta^{d g}\right)$. These $A(t)$ form a family of $d^{g}-(d g-g+1)$-dimensional subspaces of $\mathbf{C}^{d^{g}}$ that vary holomorphically with $t$. Their orthogonal complements $A(t)^{\perp}=\{c \in$ $\mathrm{C}^{d^{g}}:{ }^{t} c \cdot a=0$ for all $\left.a \in A(t)\right\}$ form a family of $d g-g+1$-dimensional subspaces of $\mathbf{C}^{d^{g}}$ that vary holomorphically with $t$. Analogous to Proposition 7 and its corollary, they have the property that

$$
A(t+d \lambda)^{\perp}=A(t)^{\perp}
$$

for any $\lambda \in \mathscr{L}$. This says we can define a vector bundle

$$
E(\mathscr{B})=\coprod_{t \in J} A(d t)^{\perp} \subset J \times \mathbf{C}^{d^{g}}
$$


of rank $d g-g+1$. Note $E(\mathscr{B})$ is a subvariety of dimension $d g+1$. Let $B=\bigcup_{t \in J} A(d t)^{\perp} \subset \mathbf{C}^{g}$. Denote $p_{2}: J \times \mathbf{C}^{g} \rightarrow \mathbf{C}^{g}$ the projection onto the second factor. Since $p_{2}$ is a proper map and $B=p_{2}(E(\mathscr{B}))$, then $B$ is an analytic subvariety of $C^{d^{g}}$ of dimension at most $d g+1$. Since $B$ is the union of linear subspaces, it is a cone, that is, $b \in B$ implies $\mathbf{C} b \subset B$. This allows us to consider its projectivization $\mathbf{P} B \subset \mathbf{C P}^{d^{8}-1}$, a subvariety of dimension $\operatorname{dim} B-1 \leq d g$. The generic linear subvariety of $\mathbf{C P}^{d^{g}-1}$ of dimension $d^{g}-$ $1-d g-1$ will not intersect $\mathbf{P} B$. Let such a linear subvariety be $\mathbf{P} W \subset \mathbf{C P}^{d^{g}-1}$ where $W \subset \mathbf{C}^{d^{g}}$ is of dimension $d^{g}-1-d g$. The fact that $\mathbf{P} W \cap \mathbf{P} B=\varnothing$ implies that $W \cap B=(0)$. Since $W^{\perp}$ is a dimension $d g+1$ subspace, we can choose a basis $c_{1}, \ldots, c_{d g+1}$ of $W^{\perp}$, for some $c_{i} \in \mathbf{C}^{d^{8}}$.

Since $B=\bigcup_{t \in \mathbf{C}^{8}} A(t)^{\perp}$ and $W \cap B=(0)$, then $W \cap A(t)^{\perp}=(0)$ for any fixed $t \in \mathbf{C}^{g}$. This implies $W^{\perp}+A(t)=\mathbf{C}^{d^{g}}$ by Proposition 8. Since

$$
\operatorname{Span}\left\{\theta_{d}\left\langle c_{i} \mid d r-t\right\rangle(w(z))\right\}_{i=1, \ldots, d g+1}=\left\{\theta_{d}\langle c \mid d r-t\rangle(w(z)): c \in W^{\perp}\right\}
$$

and

$$
\theta_{d}\langle a \mid d r-t\rangle(w(z)) \equiv 0 \quad \text { for all } a \in A(t)
$$

then

$$
\begin{aligned}
\operatorname{Span}\left\{\theta_{d}\left\langle c_{i}\right|\right. & d r-t\rangle(w(z))\}_{i=1, \ldots, d g+1} \\
& =\left\{\theta_{d}\langle c+a \mid d r-t\rangle(w(z)): c+a \in W^{\perp}+A(t)=\mathbf{C}^{d^{g}}\right\} \\
& =\Gamma\left(M, \rho_{t} \zeta^{d g}\right) .
\end{aligned}
$$

Thus for any $t \in \mathbf{C}^{g}$, the functions $\left\{\theta_{d}\left\langle c_{i} \mid d r-t\right\rangle(w(z))\right\}_{i=1, \ldots, d g+1}$ span $\Gamma\left(M, \rho_{t} \zeta^{d g}\right)$ as functions of $z$. Hence these $c_{1}, \ldots, c_{d g+1}$ are exactly what we wanted. This completes the proof of part (1).

(2) We have to show that it is not possible with any $d g$ vectors $c_{1}, \ldots, c_{d g} \in$ $\mathrm{C}^{d^{g}}$. We consider the map $m_{d}: J \stackrel{\times d}{\rightarrow} J$, multiplication by $d$.

We will denote the pullback of any bundle $\eta$ over $J$ via $m_{d}$ by $\hat{\eta}=m_{d}^{*} \eta$. The induced cohomology ring homomorphism $m_{d}^{*}: H^{*}(J, \mathbf{Z}) \rightarrow H^{*}(J, \mathbf{Z})$ is given by $m_{d}^{*} x_{i}=d x_{i}$ and $m_{d}^{*} y_{i}=d y_{i}$. In particular, we have $m_{d}^{*} \theta=$ $d^{2} \theta$. Analogous to Proposition 4, for each nonzero $c \in \mathbf{C}^{d^{8}}$, we can define a line bundle $\mu_{c}^{*}$ by $E\left(\mu_{c}^{*}\right)=\bigsqcup_{t \in J} \operatorname{Span}\left\{\theta_{d}\langle c \mid d r-d t\rangle(w)\right\} \subset E\left(\widehat{\chi^{*}}\right)=$ $\bigsqcup_{t \in J} \Gamma\left(J, \rho_{d t-d r} \xi^{d}\right)$. The Chern class of this bundle is $c\left(\mu_{c}^{*}\right)=1-d \theta$.

We have $E\left(\chi^{d g-g+1^{*}}\right)=\bigsqcup_{t \in J} \Gamma\left(M, \rho_{t} \zeta^{d g}\right)$ and $c\left(\chi^{d g-g+1^{*}}\right)=e^{-\theta}$. Let $n=d g-g+1=\operatorname{rank} \chi^{d g-g+1^{*}}$ throughout the remainder of this proof, so $E\left(\widehat{\chi^{n^{*}}}\right)=\amalg_{t \in J} \Gamma\left(M, \rho_{d t} \zeta^{d g}\right)$ and $c\left(\widehat{\chi^{n^{*}}}\right)=e^{-d^{2} \theta}$.

Suppose by contradiction that there exist $c_{1}, \ldots, c_{d g} \in \mathbf{C}^{d^{g}}$ such that 
$\left\{\theta_{d}\left\langle c_{i} \mid d r-d t\right\rangle(w(z))\right\}_{i=1}^{d^{g}}$ spans $\Gamma\left(M, \rho_{d t} \zeta^{d g}\right)$ for all $t$. This means that

$$
E\left(\mu^{*}\right)_{t}=\operatorname{Span}\left\{\theta_{d}\left\langle c_{i} \mid d r-d t\right\rangle(w(z))\right\}_{i=1}^{d g} \rightarrow E\left(\widehat{\chi^{n *}}\right)_{t}=\Gamma\left(M, \rho_{d t} \zeta^{d g}\right)
$$

is surjective, where we denote $\mu^{*}=\mu_{c_{1}}^{*} \oplus \cdots \oplus \mu_{c_{d g}}^{*}$ for simplicity. This means that $\mu^{*} \rightarrow \widehat{\chi^{n^{*}}}$ is surjective on each fiber, and so is a surjective map of vector bundles $J$. Equivalently, this means that the map of the duals, $\widehat{\chi}^{n} \rightarrow \mu$ is an injective map. We have an exact sequence

$$
0 \rightarrow \widehat{\chi^{n}} \rightarrow \mu \rightarrow Q \rightarrow 0
$$

of vector bundles, where $Q$ is the quotient bundle. Since $Q$ has rank $d g-$ $(d g-g+1)=g-1$, its $g$ th Chern class must be zero. Using the fact that $c(\mu)=(1+d \theta)^{d g}$ and $c\left(\widehat{\chi^{n}}\right)=e^{d^{2} \theta}$, we compute the Chern class of $Q$ :

$$
c(Q)=c(\mu) / c\left(\widehat{\chi^{n}}\right)=(1+d \theta)^{d g} / e^{d^{2} \theta}=(1+d \theta)^{d g} e^{-d^{2} \theta} .
$$

Thus the $g$ th Chern class of $Q$ is

$$
\begin{aligned}
c_{g}(Q) & =\sum_{j+k=g}\left(\begin{array}{c}
d g \\
j
\end{array}\right)(d \theta)^{j} \cdot \frac{\left(-d^{2} \theta\right)^{k}}{k !} \\
& =(d \theta)^{g} \sum_{j+k=g}\left(\begin{array}{c}
d g \\
j
\end{array}\right) \frac{(-d)^{k}}{k !} \\
& =(d \theta)^{g} \sum_{k=0}^{g}\left(\begin{array}{c}
d g \\
g-k
\end{array}\right) \frac{(-d)^{k}}{k !} \\
& =(d \theta)^{g} \sum_{k=0}^{g} \frac{(d g) !}{(g-k) !(d g-g+k) !} \frac{(-d)^{k}}{k !} \\
& =\frac{(d \theta)^{g}}{g !} \sum_{k=0}^{g} \frac{(d g) !}{(d g-g+k) !} \frac{g !}{(g-k) ! k !}(-d)^{k} \\
& =\frac{(d \theta)^{g}}{g !} \sum_{k=0}^{g} \frac{(d g) !}{(d g-g+k) !}\left(\begin{array}{l}
g \\
k
\end{array}\right)(-d)^{k} .
\end{aligned}
$$

Since $c_{g}(Q)=0$ and $\frac{(d \theta)^{g}}{g !} \neq 0 \in H^{2 g}(J, \mathbf{Z}) \cong \mathbf{Z}$, we must have that

$$
\sum_{k=0}^{g} \frac{(d g) !}{(d g-g+k) !}\left(\begin{array}{l}
g \\
k
\end{array}\right)(-d)^{k}=0 \text {. }
$$

Separating out the terms for $k=g$ and $k=g-1$, we get

$$
(-d)^{g}+d g^{2}(-d)^{g-1}+\sum_{k=0}^{g-2} \frac{(d g) !}{(d g-g+k) !}\left(\begin{array}{l}
g \\
k
\end{array}\right)(-d)^{k}=0 .
$$

For $k=0,1, \ldots, g-2$, we have that $d g-g+k \leq d g-2$ and so

$$
(d g-1) \text { divides } \frac{(d g) !}{(d g-g+k) !} .
$$


This implies

$$
(d g-1) \text { divides } \sum_{k=0}^{g-2} \frac{(d g) !}{(d g-g+k) !}\left(\begin{array}{l}
g \\
k
\end{array}\right)(-d)^{k},
$$

and so $(d g-1)$ divides $(-d)^{g}+d g^{2}(-d)^{g-1}$, which is the same as

$$
(d g-1) \text { divides }(-d)^{g-1}\left(d g^{2}-d\right) \text {. }
$$

Since $(d g-1)$ and $d$ are relatively prime, this implies

$$
(d g-1) \text { divides }\left(d g^{2}-d\right) \text {. }
$$

Since $d g^{2}-d=g(d g-1)+g-d$, it must be that

$$
(d g-1) \text { divides }(g-d) \text {. }
$$

Since we are assuming $g>1$ and $d>2$, we have that

$$
|g-d|<\max (g, d)-1<d g-1 .
$$

So the only way that $(d g-1)$ can divide $(g-d)$ is if $(g-d)=0$, which is $g=d$. We now consider two cases, according to whether $d$ is even or odd, and show in each case that we arrive at a contradiction.

First, we take the case where $d=g$ is odd. So $d \geq 3$. For $k=0,1, \ldots, g-$ 3 , we have that

$$
(d g-2) \text { divides } \frac{(d g) !}{(d g-g+k) !} .
$$

By equation (16), this implies that

$$
(d g-2) \text { divides }(-d)^{g}+d g^{2}(-d)^{g-1}+\frac{g(g-1)}{2} d g(d g-1)(-d)^{g-2} \text {. }
$$

Using $d=g$, we can rewrite this as

$$
\left(d^{2}-2\right) \text { divides }(-d)^{d}\left(1-d^{2}\right)+\frac{g(g-1)}{2}(-d)^{d}\left(d^{2}-1\right),
$$

or

$$
\left(d^{2}-2\right) \text { divides }(-d)^{d}\left(d^{2}-1\right)\left(-1+\frac{g(g-1)}{2}\right) .
$$

That $\left(d^{2}-2\right)$ and $\left(d^{2}-1\right)$ are relatively prime is clear. Also $\operatorname{gcd}\left(d^{2}-2, d\right)=$ $\operatorname{gcd}(-2, d)=1$ since $d$ is odd. This forces

$$
\left(d^{2}-2\right) \text { divides }-1+\frac{d(d-1)}{2},
$$

which is impossible since $-1+\frac{d(d-1)}{2}=\frac{1}{2}(d-2)(d+1)$ is positive and yet

$$
-1+\frac{d(d-1)}{2}=\frac{1}{2} d^{2}-\frac{1}{2} d-1<d^{2}-2,
$$

so we have a contradiction here. 
We now take the second case where $d=g$ is even. So $d \geq 4$. For $k=$ $0,1, \ldots, g-4$, we have that

$$
(d g-3) \text { divides } \frac{(d g) !}{(d g-g+k) !} .
$$

By equation (16), this implies that

$$
\begin{gathered}
(d g-3) \text { divides }(-d)^{g}+d g^{2}(-d)^{g-1}+\frac{g(g-1)}{2} d g(d g-1)(-d)^{g-2} \\
+\frac{g(g-1)(g-2)}{6} d g(d g-1)(d g-2)(-d)^{g-3}
\end{gathered}
$$

Using $d=g$, and after some manipulations, we can rewrite the right-hand side as

$$
\begin{aligned}
(-d)^{d} & \left(d^{2}-1\right)\left(-1+\frac{d(d-1)}{2}\right)-(-d)^{d}\left(d^{2}-1\right) \frac{(d-1)(d-2)}{6}\left(d^{2}-2\right) \\
& =\frac{1}{6}(-d)^{d}\left(d^{2}-1\right)\left[\left(d^{2}-3\right)\left(2-d^{2}+3 d-2\right)-2\right] \\
& =-(-d)^{d-1}\left(\begin{array}{c}
d+1 \\
3
\end{array}\right)\left(d^{2}-3\right)\left(-d^{2}+3 d\right)-\frac{1}{3}(-d)^{d}\left(d^{2}-1\right)
\end{aligned}
$$

This implies that

$$
\left(d^{2}-3\right) \text { divides } \frac{1}{3}(-d)^{d}\left(d^{2}-1\right) .
$$

Since $d$ is even, we have $\operatorname{gcd}\left(d^{2}-3, d^{2}-1\right)=\operatorname{gcd}\left(-2, d^{2}-1\right)=1$ so that it must be that

$$
\left(d^{2}-3\right) \text { divides }(-d)^{d} \text {. }
$$

Since $\operatorname{gcd}\left(d^{2}-3, d\right)=\operatorname{gcd}(-3, d)$, the only possible prime that divides both $\left(d^{2}-3\right)$ and $d^{d}$ is 3 . This forces

$$
\left(d^{2}-3\right)=\text { a power of } 3 \text {. }
$$

Since $d \geq 4$, we have $d^{2}-3>3^{2}$ and so necessarily $3^{2}$ divides $\left(d^{2}-3\right)$. Then 3 also divides $d$. Let $d=3 N$, for some integer $N$. But

$$
d^{2}-3=(3 N)^{2}-3=3\left(3 N^{2}-1\right)
$$

implies 3 divides $\left(3 N^{2}-1\right)$ which is clearly impossible.

Having arrived at a contradiction in each of the two cases, the proof is now complete.

Proof of Theorem 5. Denote the base space by $X$. We are given that there is an injection

$$
E(\mathscr{A}) \hookrightarrow E\left(\mathscr{I}_{N}\right)=X \times \mathbf{C}^{N} .
$$

(1) Let $p_{2}: E\left(\mathscr{I}_{N}\right)=X \times \mathbf{C}^{N} \rightarrow \mathbf{C}^{N}$ be the projection onto the second factor, and let $R=p_{2}(E(\mathscr{A}))$. The fact that $\mathscr{A}$ is bundle of rank $n$ over an analytic variety of dimension $k$ implies that $E(\mathscr{A})$ is an analytic variety of dimension $k+n$. Since $X$ is compact, $p_{2}$ is clearly a proper map, so $R$ is an anaytic subvariety of $\mathbf{C}^{N}$ of dimension at most $k+n$. The property that $R$ is a 
cone allows us to consider its projectivization $\mathbf{P} R$ as an analytic subvariety of $\mathbf{C P}^{N-1}$ of dimension $\operatorname{dim} R-1$. The fact that $\mathbf{P} R$ has dimension less than or equal to $n+k-1$ implies that a generic linear subspace of $\mathbf{C} \mathbf{P}^{N-1}$ of dimension $N-1-(n+k)$ will not intersect $\mathbf{P} R$. Pick some generic linear subspace $K \subset \mathbf{C}^{N}$ of dimension $N-(n+k)$, so $\mathbf{P} K \subset \mathbf{C P}^{N-1}$ will be of dimension $N-1-(n+k)$ and thus will not intersect $\mathbf{P} R$. This implies that $K \cap R=(0)$. Consider the canonical projection map $\pi: \mathbf{C}^{N} \rightarrow \mathbf{C}^{N} / K$. Fix an identification $\mathbf{C}^{N} / K=\mathbf{C}^{n+k}$. This gives a map of vector bundles $\mathscr{I}_{N} \rightarrow \mathscr{I}_{n+k}$ by

$$
E\left(\mathscr{I}_{N}\right)=X \times \mathbf{C}^{N} \stackrel{1 \times \pi}{\longrightarrow} E\left(\mathscr{I}_{n+k}\right)=X \times \mathbf{C}^{n+k},
$$

whose kernel is $X \times K$. We are interested in the composition $\mathscr{A} \hookrightarrow \mathscr{I}_{N} \rightarrow \mathscr{I}_{n+k}$. On each fiber over $x \in X$, the map is

$$
E(\mathscr{A})_{x}=R \cap E\left(\mathscr{I}_{N}\right)_{x} \rightarrow E\left(\mathscr{I}_{n+k}\right)_{x},
$$

The kernel is $(X \times K) \cap\left(R \cap E\left(\mathscr{I}_{N}\right)_{x}\right)=(K \cap R) \cap E\left(\mathscr{I}_{N}\right)_{x}=(0)$, so the map is injective on each fiber. This gives us an injection $\mathscr{A} \hookrightarrow \mathscr{I}_{n+k}$, which completes the proof of part (1).

(2) We now assume in addition that the $k$ th Segre class of $\mathscr{A}, s_{k}(\mathscr{A}) \in$ $H^{2 k}(X, \mathbf{Z})$, is nonzero. Recall that the Segre class is defined as the inverse of the Chern class. Precisely, we have $s(\mathscr{A})=1 / c(\mathscr{A}) \in H^{*}(X, \mathbf{Z})$.

Suppose by contradiction that $n+k$ is not the minimum number required, so there exists some imbedding $\mathscr{A} \hookrightarrow \mathscr{I}_{m}$ for some $m \leq n+k-1$. There is an exact sequence

$$
0 \rightarrow \mathscr{A} \rightarrow \mathscr{I}_{m} \rightarrow Q \rightarrow 0
$$

of vector bundles over $X$, where $Q$ is the quotient bundle. The fact that $\operatorname{rank} Q=m-n \leq k-1$ implies that the $k$ th Chern class of $Q, c_{k}(Q)$, must be zero. But we have that

$$
c(Q)=c\left(\mathscr{I}_{m}\right) / c(\mathscr{A})=1 / c(\mathscr{A})=s(\mathscr{A}),
$$

so that $c_{k}(Q)=s_{k}(\mathscr{A})$ is nonzero by assumption. This contradiction completes the proof.

In fact the construction of the imbedding $\mathscr{A} \hookrightarrow \mathscr{I}_{n+k}$ done in the proof above says more.

Corollary 1. Suppose we have the same assumptions as in Theorem 5. Then there exists an imbedding $\mathscr{A} \hookrightarrow \mathscr{I}_{n+k}$ where $\mathscr{I}_{n+k}$ is some quotient bundle of $\mathscr{I}_{N}$ and the imbedding comes from the composition $\mathscr{A} \hookrightarrow \mathscr{I}_{N} \rightarrow \mathscr{I}_{n+k}$.

The dual statement of this corollary is the following.

Corollary 2. Let $\mathscr{A}$ be a (holomorphic) vector bundle of rank $n$ over a compact analytic variety of dimension $k$. Suppose there is a surjection $\mathscr{I}_{N} \rightarrow \mathscr{A}$, for some $N \geq n+k$. Then:

(1) There exists a subbundle $\mathscr{I}_{n+k} \hookrightarrow \mathscr{I}_{N}$, so that the restricted map $\mathscr{I}_{n+k} \rightarrow \mathscr{A}$ remains surjective. 
(2) If in addition the kth Segre class of $\mathscr{A}, s_{k}(\mathscr{A})$, is nonzero, then $n+k$ is the minimum number necessary.

\section{FinAl REMARKS}

We will illustrate another usage of the identification $M^{[n]}=\mathbf{P} E\left(\chi^{n-g+1^{*}}\right)$. In [16], Mattuck was interested in cross sections of the projective bundle $M^{[n]} \rightarrow$ $J$, with $n \geq 2 g-1$. There he proved that $M^{[2 g-1]} \rightarrow J$ has no cross sections for "general" $M$, but that for $n>4 g, M^{[n]} \rightarrow J$ does have cross sections. Observe that for any vector bundle $\mathscr{A}$, a cross section of $\mathbf{P} \mathscr{A}$ corresponds exactly to a line subbundle of $\mathscr{A}$. The fact that $M^{[2 g-1]}$ has no cross sections is equivalent to the fact that $\chi^{g *}$ has no line subbundles, which can be deduced as follows. Suppose $\chi^{g *}$ has a line subbundle, call it $L$. For "general" $M$, the codimension 1 divisors of $J(M)$ are all linear combinations of translations of the theta divisor, so necessarily $c(L)=1+a \theta$ for some $a \in \mathbf{Z}$. Then $c\left(\chi^{g *} / L\right)=c\left(\chi^{g}\right) / c(L)=e^{-\theta} /(1+a \theta)=e^{-\theta}\left(1-a \theta+a^{2} \theta^{2}-\cdots\right)$. But $\operatorname{rank} \chi^{g *} / L=g-1$ implies

$$
0=c_{g}\left(\chi^{g^{*}} / L\right)=(-\theta)^{g}\left[a^{g}+a^{g-1}+\frac{a^{g-2}}{2 !}+\cdots+\frac{1}{g !}\right],
$$

which contradicts the fact that the exponential polynomial has no rational roots. This was essentially the proof Mattuck gave.

We can improve on his bound of $4 g$ by the following argument. Consider the bundle $\chi^{g+1^{*}}$. Since $\xi$ is an ample line bundle on $J$, we have for some sufficiently large $N \in \mathbf{Z}$ that $\xi^{N} \otimes \chi^{g+1^{*}}$ is generated by global sections. The rank of this bundle is 1 larger than the dimension of the base space. By Atiyah's theorem [3, Theorem 2], $\xi^{N} \otimes \chi^{g+1^{*}}$ has a trivial line subbundle, $\mathscr{I} \hookrightarrow \xi^{N} \otimes$ $\chi^{g+1^{*}}$. Thus we have $\xi^{-N} \hookrightarrow \chi^{g+1^{*}}$, so that $\chi^{g+1^{*}}$ has a line subbundle. This means that $M^{[2 g]}$ has a cross section.

Theorem 6. The projective bundle $M^{[n]} \rightarrow J$ has cross sections for $n \geq 2 g$.

So we obtain an imbedding $J \hookrightarrow M^{[2 g]}$. In fact, being a cross section, this is a nonsingular imbedding.

This theorem on the existence of cross sections of projective bundles can be generalized to more general varieties.

Theorem 7. Let $X$ be a nonsingular projective variety of dimension $k$. Then any projective bundle $P$ of dimension $n \geq k$ has a cross section.

Proof. We will use Serre's results [18] that over a projective variety, every coherent analytic sheave corresponds uniquely to a coherent algebraic sheave. So for the moment, view $P$ as an algebraic projective bundle. Since $X$ is nonsingular, in particular locally factorial, we may apply a result of Grothendieck $[5, \S 3.4]$ that every algebraic projective bundle is derived from some algebraic vector bundle. Let $E$ be some vector bundle of rank $n+1$ for which $P=\mathbf{P} E$. 
Being a projective variety, $X$ has an ample line bundle. Let $\xi$ be an ample line bundle on $X$. For some large enough $N, \xi^{N} \otimes E$ is generated by global sections. Since this vector bundle has dimension $n+1>k$, then by Atiyah's theorem [3, Theorem 2], it has a trivial subbundle $\mathscr{I} \hookrightarrow \xi^{N} \otimes E$. Then $\xi^{-N} \hookrightarrow E$ is a line subbundle of $E$. This implies that $P$ has a cross section.

In particular, since the Jacobian of a curve is a nonsingular projective variety, we have

Corollary. Every projective bundle over $J$ of dimension greater than $g$ has a cross section.

\section{REFERENCES}

1. E. Arbarello, Fay's trisecant formula and a characterization of Jacobian varieties, Proc. Sympos. Pure Math., vol. 46, Amer. Math. Soc., Providence, R.I., 1987, pp. 49-61.

2. E. Arbarello, M. Cornalba, P. A. Griffiths, and J. Harris, Geometry of algebraic curves, Volume I, Springer-Verlag, 1985.

3. M. F. Atiyah, Vector bundles over an elliptic curve, Proc. London Math. Soc. (3) 7 (1957), 414-452.

4. W. Fulton, Intersection theory, Springer-Verlag, 1984.

5. A. Grothendieck, Sur quelques points d'algèbre homologique, Tôhoku Math. J. (2) 9 (1957), 119-221.

6. R. C. Gunning, On generalized theta functions, Amer. J. Math. 104 (1982), 183-208.

7. _ Riemann surfaces and generalized theta functions, Springer-Verlag, 1976.

8. __ Some curves in Abelian varieties, Invent. Math. 66 (1982), 377-389.

9. R. Hartshorne, Algebraic geometry, Springer-Verlag, 1977.

10. F. Hirzebruch, Topological methods in algebraic geometry, Springer-Verlag, 1966.

11. D. Husemoller, Fibre bundles, McGraw-Hill, 1966.

12. G. Kempf, Inversion of Abelian integrals, Bull. Amer. Math. Soc. (N.S.) 6 (1982).

13. $\ldots$, On the geometry of a theorem of Riemann, Ann. of Math. 98 (1973).

14. J. L. Koszul, Multiplicateurs et classes caractéristiques, Trans. Amer. Math. Soc. 89 (1958), 256-266.

15. H. H. Martens, On the varieties of special divisors on a curve, J. Reine Angew. Math. 227 (1968), 111-120.

16. A. Mattuck, Picard bundles, Illinois J. Math. 5 (1961), 550-564.

17. __ Symmetric products and Jacobians, Amer. J. Math. 83 (1961), 189-206.

18. J.-P. Serre, Géométrie analytique et géométrie algébrique, Ann. Inst. Fourier (Grenoble) 4 (1955-56), 1-42.

Department of Mathematics, University of Rochester, Rochester, New York 14627

E-mail: DYUE@UHURA.CC.ROCHESTER.EDU 